\title{
Multiconnectivity for Mobility Robustness in Standalone 5G Ultra Dense Networks with Intrafrequency Cloud Radio Access
}

\author{
Fasil B. Tesema, ${ }^{1}$ Ahmad Awada, ${ }^{1}$ Ingo Viering, ${ }^{2}$ \\ Meryem Simsek, ${ }^{3}$ and Gerhard P. Fettweis ${ }^{3}$ \\ ${ }^{1}$ Nokia Bell Labs, 81541 Munich, Germany \\ ${ }^{2}$ Nomor Research GmbH, 81541 Munich, Germany \\ ${ }^{3}$ Technische Universität Dresden, Vodafone Stiftungslehrstuhl, 01062 Dresden, Germany \\ Correspondence should be addressed to Fasil B. Tesema; fasil.berhanu_tesema.ext@nokia.com
}

Received 15 June 2016; Accepted 18 August 2016; Published 9 January 2017

Academic Editor: Yoshikazu Miyanaga

Copyright (C) 2017 Fasil B. Tesema et al. This is an open access article distributed under the Creative Commons Attribution License, which permits unrestricted use, distribution, and reproduction in any medium, provided the original work is properly cited.

\begin{abstract}
Capacity and ultra-reliable communication are some of the requirements for 5th generation (5G) networks. One of the candidate technologies to satisfy capacity requirement is standalone Ultra Dense Network (UDN). However, UDNs are characterized by fast change of received signal strength that creates mobility challenges in terms of increased handovers and connection failures. In this paper, a low layer multiconnectivity scheme is presented for standalone UDN aiming at ultra-reliable communication that is free of interruptions from handover procedures and connection failures. Furthermore, the problem in managing of the set of serving cells, that are involved in multiconnectivity for each user, is formulated. By using numerical method, feasible scheme for management of the set of serving cells is derived. Performance of the proposed multiconnectivity scheme is evaluated and compared against single connectivity. It is shown that the proposed multiconnectivity scheme outperforms single connectivity considerably in terms of connection failures and cell-edge throughput.
\end{abstract}

\section{Introduction}

Mobile communication technology has evolved very fast over the past few years to cope with sophisticated device technologies and diverse applications. The evolution is expected to continue in order to adapt to the societal changes of future information society. One of the projects that are established targeting 5G networks is mobile and wireless communications enablers for the twenty-twenty information society (METIS) [1]. The goal of the METIS project is to lay the foundation for the beyond $20205 \mathrm{G}$ mobile and wireless system. The work in [1-3] elaborates the challenges, requirements, and candidate technologies of the mobile networks in the future. Some of the requirements are increased capacity and ultra-reliable communication. For example, capacity and ultra-reliable communication are required to satisfy such applications as real-time remote computing. Real-time remote computing is demonstrated as a test case in [4] where mobile users require high data rate without interruption.
One of the key enablers to achieve high capacity is Ultra Dense Network (UDN). One use case of UDN is to have dense small cells with centralized processing [5]. Centralized processing enables efficient radio resource management across multiple cells without exchange of signaling among cells. Moreover, the centralized processing can be implemented as cloud technology, by using Network Function Virtualization (NFV) and Software Defined Networking (SDN). NFV and SDN are considered by some mobile vendors and operators as a candidate technology for 5G Radio Access Network (RAN) [6-8]. Besides, optical fiber can be used as the physical link between the central processing node and the access point in order to give the ideal backhaul media with sufficient bandwidth. The concept of transmission of a radio over fiber is called "radio over fiber" and a use case for cloud architecture is demonstrated in [9].

Standalone UDNs are characterized by fast change of received signal. The fast change of the signal creates mobility 


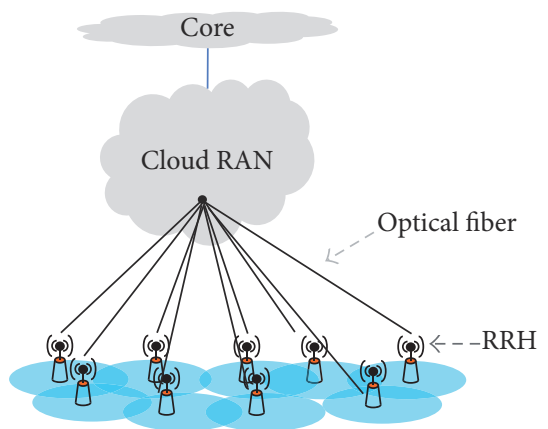

(a)

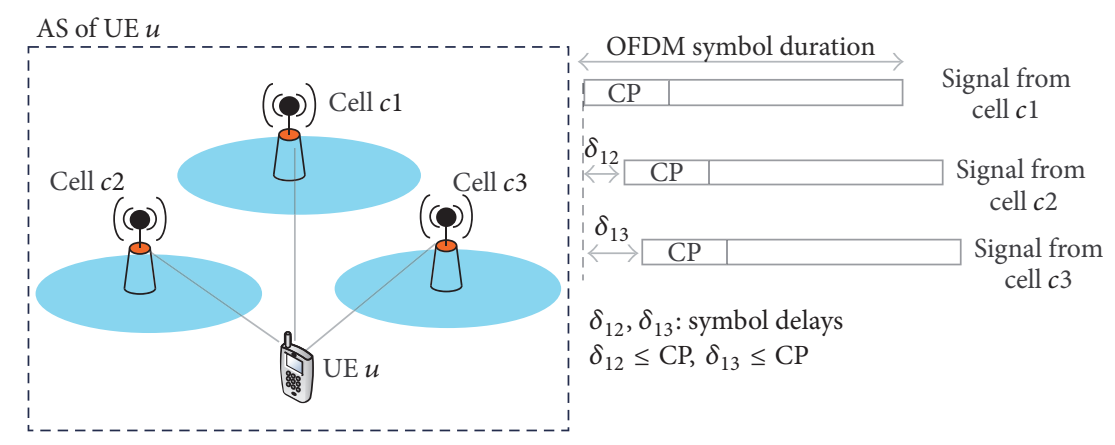

(b)

FIGURE 1: A cloud RAN with RRHs in (a) having only RF functionalities connected via optical fiber to a central unit that processes basic RAN functionalities such as PHY, MAC, radio resource management, and admission control and example of ISI free SFN transmission in (b) for UE with an AS of 3 cells.

challenges, such as high number of handovers, and connection failures, for example, handover failures and Radio Link Failures (RLFs), that result in service interruption. As previously mentioned, one of the requirements is that high data rates are delivered to users without service interruptions. Such provisioning can be achieved by a scheme that ensures ultra-reliable communication that is free of connection failures and by a scheme that prepares the serving cell(s) before the transmission is broken. In this work, the envisioned scheme for achieving the aforementioned targets is referred to as "multiconnectivity" and it focuses on intrafrequency transmissions.

Multiconnectivity as a general concept is not a new topic. Some of the multiconnectivity solutions in Long Term Evolution (LTE) are intersite Carrier Aggregation (CA), Dual Connectivity (DC), and Coordinated Multipoint (CoMP) transmission. CA is a key feature in LTE and creates a larger capacity by combining separate intraband or interband carrier components [10-12]. CA was first introduced in LTE Release 10 for aggregation of component carriers per one site; intersite CA was later introduced in LTE Release 11.

DC has been proposed in LTE Release 12 as one of the features for small cell enhancements $[13,14]$. According to [13], DC is the operation where a User Equipment (UE) consumes radio resources provided by at least two different network points connected with nonideal backhaul. However, current solutions of DC focus on Heterogeneous Network (HetNet), for example, Macro and Pico base stations. While capacity can be achieved through dense deployment of cells, the small cells are restricted to be deployed inside the coverage area of the Macro cells.

The other multiconnectivity feature in LTE is CoMP which is designed for intrafrequency transmissions and primarily targets enhancement of the throughput of cell-edge users $[15,16]$. Herein, the mobility robustness is handled by only one cell which is termed as Primary Cell (PCell). Consequently, the mobility challenge is equivalent to single connectivity because a UE purely depends on the link to the PCell which is changed by a conventional handover.

\section{Previous Work and Contribution}

This paper focuses primarily on mobility robustness in standalone UDN. The architecture assumption is a cloud RAN architecture, shown in Figure 1(a), where access points with only Radio Frequency (RF) functionality, similar to Remote Radio Heads (RRHs), are connected via fiber to a central unit that processes the basic RAN functionalities such as physical (PHY), Media Access Control (MAC), admission control, and radio resource management. However, the principles can be extended to other architecture assumptions. The access points in this work are referred to as "cell" which is used as a general expression for an antenna array that is mounted on a single site and having only RF functionalities. Due to complexity, this work addresses only the downlink transmission of signals in a network of cells managed by one cloud.

Our previous work on modeling and evaluation of a low layer multiconnectivity scheme that uses Single Frequency Network (SFN) transmission is discussed in [17]. The term SFN refers to noncoherent joint transmission of a signal on the same radio resource in frequency and time. The downlink multiplexing scheme assumed is Orthogonal Frequency Division Multiplexing (OFDM) and it allows a synchronous multicell transmission. The set of cells that are prepared for coordinated transmission to a UE are termed as Active Set (AS). With such a transmission scheme, a UE receives multiple copies of the same signal with different propagation delays from the cells in its AS. The UE receiver can constructively combine these multiple copies provided that they are received within the duration of cyclic prefix (CP) which is attached at the beginning of each OFDM symbol. Otherwise, intersymbol interference (ISI) occurs as the symbol received with higher delay overlaps with the subsequent symbol from a transmitting station with much smaller delay. An example is given in Figure 1(b) which shows an AS of 3 cells transmitting synchronously to UE $u$ and their signals arriving within $\mathrm{CP}$ duration.

In [17], it is assumed that SFN transmission is performed not only for data signals but also for control signals. It is 
shown that the gain in signal quality on the control and data signals, due to SFN transmission, improves mobility robustness and throughput of cell-edge UE. Herein, this paper adds the following contributions on top of the previous work:

(i) The problem in AS management is formulated considering the uncertainty in UE measurement with the target of providing ultra-reliable communication. UE measurements are needed to support the network in AS management. Instantaneous UE measurements are prone to unstable AS management decisions due to fluctuations caused by noise and fast fading. Thus, the UE measurement should be processed properly to create stable decisions. Moreover, procedures in AS management should ensure that strongest cell(s) are included in the AS to achieve ultra-reliable communication that is free of connection failures. Thus, the problem in AS management is formulated taking the aforementioned details into account.

(ii) A feasible AS management scheme is derived by using numerical method taking an elaborated mobility scenario into account. One of the solutions to avoid the fluctuations in UE measurements is averaging the instantaneous UE measurements. However, averaging the UE measurements induces delay on AS management decisions. Derivation of AS management schemes by using analytical methods that take into account the delay from UE measurement averaging and detailed aspects of the network, such as distance dependent path loss, shadowing, fast fading, noise, and real movement of UE pieces, is not straight forward. Consequently, a feasible AS management scheme is derived by using numerical method.

Furthermore, one of the practical implementations of the derived AS management scheme is explained with support of prior art AS management procedures. The practical implementation of the AS management scheme is based on averaged UE measurement.

(iii) The performance of the practical implementation of the AS management scheme is compared with an upper bound performance that assumes ideal UE measurement that is free of fluctuations and delay. The upper bound performance is used as the baseline to evaluate the derived AS management scheme in terms of connection failures.

(iv) Comprehensive evaluation is performed on the interaction of key parameters of the UE measurement and the practical AS management scheme. Some of the key parameters are averaging time constant of UE measurement, diversity order of the UE, and parameters related to including/excluding of a cell to/from an AS of a UE. Among other things, the level of connection failures and the signaling overhead associated to AS management are evaluated. Furthermore, performance of the proposed multiconnectivity scheme that uses the practical AS management is compared with that of a conventional single-connectivity solution which is discussed in Section 8.1.6.

The remainder of the paper is structured as follows. First, the system model is elaborated in Section 3. Then, the problem formulation in management of AS is presented in Section 4. In Section 5, a feasible AS management scheme is derived by using numerical method. One of the practical implementations of the derived AS management scheme is elaborated in Section 6. Moreover, the scenario and parameter settings used for numerical analysis and performance evaluation are presented in Section 7. Evaluation methodologies and simulation results are shown in Section 8. Finally, concluding remarks are presented in Section 9.

\section{System Model}

This section presents the models for UE measurements, downlink Signal to Interference plus Noise Ratio (SINR) calculation, resource allocation and throughput calculation in SFN transmission, and RLF.

3.1. UE Measurement Model. For proper management of serving cell(s) for a UE, the UE sends to the network cell measurements carried out on reference symbols. This section elaborates the modeling for UE measurement with focus on measurement of power on Cell-specific Reference Symbols (CRS). Such measurement in LTE is referred to as Reference Signal Received Power (RSRP) [18] and a similar principle is assumed in this work for $5 \mathrm{G}$ network. A UE performs measurements for all detected cells. Let the set of cells that are detected by UE $u$ be denoted by $\mathscr{D}_{u}$. The measurement $M_{u, c}^{\mathrm{L}}(n)$ performed by UE $u$ for cell $c \in \mathscr{D}_{u}$ at given time step $n$ is defined in the linear domain as follows:

$$
M_{u, c}^{\mathrm{L}}(n)=P_{\mathrm{TX}} \cdot \bar{\alpha}_{u, c}^{2}(n) \cdot 10^{\left(\Omega_{u, c}(n)+\epsilon_{\text {noise }}(n)\right) / 10},
$$

where $P_{\mathrm{TX}}$ is the transmitted power on the CRS, $\epsilon_{\text {noise }}$ is the measurement error in $\mathrm{dB}$ domain, and $\bar{\alpha}_{u, c}^{2}$ is the power envelop of fast fading in linear domain. Furthermore, $\Omega_{u, c}(n)$ is the combination of the distance dependent path loss $\mu_{u, c}(n)$ and shadowing $s_{u, c}(n)$ in $\mathrm{dB}$ domain at the given position of $\mathrm{UE} u$ at time step $n$; that is,

$$
\Omega_{u, c}(n)=\mu_{u, c}(n)+s_{u, c}(n) .
$$

Expression of the measurement in $\mathrm{dB}$ domain is

$$
\begin{aligned}
M_{u, c}^{\mathrm{dB}}(n)= & 10 \cdot \log P_{\mathrm{TX}}+\Omega_{u, c}(n)+20 \cdot \log \bar{\alpha}_{u, c}(n) \\
& +\epsilon_{\text {noise }}(n) .
\end{aligned}
$$

The measurement error occurs due to noise and the limited number of reference symbols. Such error is modeled in literature as lognormal with zero mean and standard deviation $\sigma_{\epsilon}[19,20]$. It is shown in [19] that $\sigma_{\epsilon}$ decreases with higher number of reference symbols. In this work, the measurement error is modeled based on [21] as truncated lognormal distribution with limits at $3.29 \sigma_{\epsilon}$ based on worst case performance of devices. 
The model for the power envelop of fast fading $\bar{\alpha}_{u, c}^{2}(n)$ is coupled with diversity order. Fast fading accounts for the rapid fluctuations in the measurement of the UE resulting from multipath propagation [22]. The degree of fluctuation in UE measurement due to fast fading depends on the number of independent links between a receiver and a transmitter. In this paper, the number of independent links is referred to as diversity order $D$. The independent links may originate from multiple receive antennas (spatial diversity) or multiple propagation paths that are resolved at the UE receiver (frequency diversity). For a UE receiver with $D$ independent links, the fast fading power gain is the average of the independent links; that is,

$$
\bar{\alpha}_{u, c}^{2}(n)=\frac{1}{D} \sum_{i}^{D}\left|\alpha_{u, c}^{i}(n)\right|^{2},
$$

where $\alpha_{u, c}^{i}(n)$ is the amplitude of the fast fading of the individual links. The fast fading power gain $\bar{\alpha}_{u, c}^{2}(n)$ is $\chi^{2}$ distributed with $2 D$ degree of freedom [23].

3.2. Model for SINR Calculation. This section shows the underlying network assumptions and the modeling for SINR calculation. The envisioned $5 \mathrm{G}$ radio frame structure is time synchronized Time Division Duplex (TDD) with radio numerology discussed in $[24,25]$. This work focuses on mobility investigation with support of simulation that runs at higher time step granularity compared to the Transmission Time Interval (TTI). Thus, abstract models are used for SINR and throughput calculation based on principles described in $[26,27]$.

Assume a cloud network with set of cells $\mathscr{W}$ serving a set of users $\mathcal{U}$. The number of cells and the number of users in the cloud are defined as $W=|\mathscr{W}|$ and $U=|\mathscr{U}|$, respectively. Assume a UE $u \in U$ is connected to the AS $\mathscr{A}_{u}$. The data and control signals are simultaneously transmitted from all cells of the AS $\mathscr{A}_{u}$ using SFN transmission. The signal components of the cells in the same AS are assumed to fall within the cyclic prefix resulting in full combination of the power from each cell in the AS as described in [28]. Consequently, the SINR $\gamma_{u}(n)$ of UE $u$ at time step $n$ is defined as

$$
\gamma_{u}(n)=\frac{\sum_{c \in \mathscr{A}_{u}} P_{u, c}(n)}{\sum_{c \notin \mathscr{A}_{u}} \rho_{c} P_{u, c}(n)+N},
$$

where $P_{u, c}(n)$ is the signal strength received at UE $u$ from cell $c$ at time step $n, \rho_{c}$ is the fraction of resource utilization in cell $c$, and $N$ is the thermal noise power. Full buffer traffic model is assumed; thus, $\rho_{c}$ is assumed to be equal to 1 for all $c$. In this work, the definition of SINR $\gamma_{u}(n)$ given in (5) is assumed to represent the signal quality of both data and control signals.

3.3. Model for Resource Allocation and Throughput Calculation. This section presents the principles behind the models for resource allocation and throughput calculation. A centralized resource allocation scheme is designed based on the concept of proportional-fairness as demonstrated in [17].

Assume a resource fraction $0 \leq r_{u}^{\mathrm{PF}}(n) \leq 1$ of the totally available bandwidth $B$ is allocated to UE $u$ at time step $n$ based on proportional-fair scheduler. Then, the throughput $R_{u}(n)$ of $\mathrm{UE} u$ at time step $n$ is defined as

$$
R_{u}(n)=r_{u}^{\mathrm{PF}}(n) \Phi\left(\gamma_{u}(n)\right),
$$

where $\Phi\left(\gamma_{u}(n)\right)$ is the throughput of UE $u$ over the bandwidth $B$ and it is a function of the SINR $\gamma_{u}(n)$. In this work, Shannon capacity formula, which is described in $[29,30]$, is used as a mapping function from $\gamma_{u}(n)$ to $\Phi\left(\gamma_{u}(n)\right)$; that is,

$$
\Phi\left(\gamma_{u}(n)\right)=B \log _{2}\left(1+\gamma_{u}(n)\right)
$$

The SINR $\gamma_{u}(n)$ is calculated in (5) based on principles discussed in Section 3.2 and the resource fraction $r_{u}^{\mathrm{PF}}(n)$ is calculated as follows. The resource allocation model is applied at each time step; thus, the time step index $n$ is excluded in the following analysis for the sake of simplicity.

A vector of fraction of resources, $\bar{r}^{\mathrm{PF}}=\left[r_{1}^{\mathrm{PF}}, r_{2}^{\mathrm{PF}}, \ldots, r_{U}^{\mathrm{PF}}\right]$, is "proportional-fair" only if for any other feasible resource allocation $\bar{r}=\left[r_{1}, r_{2}, \ldots, r_{U}\right]$ the sum of the relative throughput gain is less than or equal to 0 ; that is,

$$
\sum_{u=1}^{U} \frac{R_{u}\left(\bar{r}^{\mathrm{PF}}\right)-R_{u}(\bar{r})}{R_{u}\left(\bar{r}^{\mathrm{PF}}\right)} \leq 0 .
$$

It is shown in [31] that fulfilling the above inequality is the same as maximizing the total utility $G$ of UE; that is,

$$
G=\sum_{u=1}^{U} \log \left(R_{u}(\bar{r})\right) .
$$

Consider column vector representations: $\bar{r}=\operatorname{col}\left\{r_{1}\right.$, $\left.r_{2}, \ldots, r_{U}\right\}$ and $\bar{\Phi}=\operatorname{col}\left\{\Phi\left(\gamma_{1}\right), \Phi\left(\gamma_{2}\right), \ldots, \Phi\left(\gamma_{U}\right)\right\}$. The utility function in (9) can be rewritten as

$$
G=\bar{r}^{T} * \bar{\Phi}
$$

where $\bar{r}^{T}$ is the transpose of $\bar{r}$ and the operator $*$ is defined as the summation of logarithm of element-wise product of two vectors. Consequently, the problem of resource allocation can be formulated as

$$
\begin{aligned}
\bar{r}^{\mathrm{PF}} & =\underset{\bar{r}}{\arg \max } \bar{r}^{T} * \bar{\Phi}, \\
\mathbf{H} \bar{r}-[1]_{W \times 1} & \leq[0]_{W \times 1}, \\
-\bar{r} & \leq[0]_{U \times 1},
\end{aligned}
$$

where $\mathbf{H}$ is $W \times U$ matrix that represents the constraint of resource allocation on each cell. For each cell, the sum of fraction of resources allocated to users that are served by the $\mathrm{AS}(\mathrm{s})$ that contains the cell can not be greater than 1; that is,

$$
\begin{gathered}
\sum_{\left\{u \mid c \in \mathscr{A}_{u}\right\}} r_{u}-1 \leq 0, \quad c=1,2, \ldots, W \\
H_{u, c}= \begin{cases}0, & \text { if } c \notin \mathscr{A}_{u} \\
1, & \text { otherwise. }\end{cases}
\end{gathered}
$$


The constraint $-\bar{r} \leq[0]_{U \times 1}$ is used to ensure that a resource allocation can not be a negative number.

There are a number of well-established existing methods to solve the aforementioned convex optimization problem. It is shown in [32] that interior-point algorithms are computationally efficient and robust for such a problem. Accordingly, interior-point algorithm from Matlab Optimization Tool is used to solve the problem.

3.4. RLF Model. Failure detection is essential in a cellular network because it avoids the fact that a UE stays too long in a bad condition. Typical example is when a moving UE is at the cell edge and the interference from a neighbor cell is too strong and causes bad link quality between the UE and a serving cell. In the absence of proper mobility decision, failure detection enables the UE to autonomously set up an alternative link by using connection reestablishment procedures. This section presents the model for RLFs based on SINR.

Similar to the notion of existing LTE-A systems [33], radio link monitoring is a technique by which a UE tracks the quality of the link to its serving cell(s). The link quality is modeled in terms of SINR given in (5). When the UE detects that the link to its serving cell(s) is weak for a certain time step $T_{\mathrm{RLF}}$, it declares the link to be in RLF. For example, the count of the time steps towards $T_{\mathrm{RLF}}$ is started when the SINR $\gamma_{u}(n)$ is less than a certain out-of-sync threshold $\mathrm{TH}_{\text {out }}$. RLF occurs at time step $n_{0}$ if the SINR $\gamma_{u}(n)$ remains below a certain insync threshold $\mathrm{TH}_{\mathrm{in}}$ for the time step interval $T_{\mathrm{RLF}}$; that is,

$$
\gamma_{u}(n)<\mathrm{TH}_{\mathrm{in}}, \quad \text { for } n_{0}-T_{\mathrm{RLF}}<n<n_{0} .
$$

The in-sync threshold $\mathrm{TH}_{\text {in }}$ should be configured to be higher than the out-of-sync threshold $\mathrm{TH}_{\text {out }}$ for stable radio link monitoring. The RLF does not occur if the SINR $\gamma_{u}(n)$ gets stronger than the in-sync threshold $\mathrm{TH}_{\text {in }}$ before time step interval $T_{\mathrm{RLF}}$ is reached. Furthermore, it is assumed that a UE in RLF attempts connection reestablishment to one of the strongest cells within a certain recovery time steps $T_{\text {recovery }}$. The connection reestablishment will be successful if the target cell for reestablishment has sufficient link quality, that is, SINR greater than a certain RLF reestablishment threshold $\mathrm{TH}_{\text {recovery}}$.

\section{Problem Formulation}

In ultra dense deployment of intrafrequency 5G wireless networks, performance is expected to be interference limited with full coverage. Interference from strong neighbor cell(s) is one of the major sources of mobility problems. Multiconnectivity helps establish links to multiple cells that are referred to as members of an AS. An AS that contains the strongest cell has less probability to be affected by RLFs that occur due to interference. For example, if the SINR threshold $\mathrm{TH}_{\text {out }}$ for RLFs is $-8 \mathrm{~dB}$ and a UE is connected to the strongest cell that exceeded the 2 nd strongest cell by $3 \mathrm{~dB}$ then more than 12 interferer cells with equal strength to 2 nd strongest cell can lead to RLFs. However, if the strongest cell is not in the AS and if it is $3 \mathrm{~dB}$ stronger than the 2 nd strongest cell serving the
UE then only 4 interferer cells with equal signal strength to the strongest cell lead to RLFs. Thus, proper AS management scheme should target including the strongest cell in the AS. This section formulates AS management problem.

The instantaneous measurement of a UE $M_{u, c}^{\mathrm{dB}}(n)$ in (3) fluctuates faster due to fast fading and noise. Consequently, instantaneous measurement leads to unstable AS configuration decisions that result in high signaling overhead. Thus, processing the measurement is required to capture the slow changing channel $Q_{u, c}(n)$ of the UE $u$; that is,

$$
Q_{u, c}(n)=10 \cdot \log P_{\mathrm{TX}}+\Omega_{u, c}(n)[\mathrm{dB}] .
$$

For a fixed UE position, the slow changing channel $Q_{u, c}(n)$ is deterministic in the sense that it depends on the path loss and shadowing condition of the UE position. However, $Q_{u, c}(n)$ is unknown to the UE and the network because $M_{u, c}(n)$ contains the fast fading and noise in addition to $Q_{u, c}(n)$.

If the statistical behavior of $Q_{u, c}(n)$ is known, the AS configuration of a UE $u$ can be based on the probability $q_{u, c}$ that a cell $c$ is the strongest for $\mathrm{UE} u$; that is,

$$
q_{u, c}=\operatorname{Pr}\left[Q_{u, c}(n)>Q_{u, c^{\prime}}(n), \forall c^{\prime} \in \mathscr{D}_{u} \wedge c^{\prime} \neq c\right] .
$$

Let $\mathscr{C}$ be the sorted list of the cells detected by UE $u$ ordered in terms of descending probabilities $q_{u, c}$; that is,

$$
\mathscr{C}=\left\{\underset{c \in \mathscr{D}_{u}}{\arg \max } q_{u, c}, \ldots, \underset{c \in \mathscr{D}_{u}}{\arg \min } q_{u, c}\right\} .
$$

For ultra-reliable communication, $\mathscr{A}_{u}$ can be formulated as a set which contains the strongest cell(s) with a certain reliability threshold, $R_{\mathrm{TH}}$; that is,

$$
\begin{aligned}
& \mathscr{A}_{u}=\{\mathscr{C}(1), \ldots, \mathscr{C}(I)\}, \\
& \text { where } \sum_{i=1}^{I} q_{u, \mathscr{C}(i)} \geq R_{\mathrm{TH}}, \quad I \leq|\mathscr{C}|
\end{aligned}
$$

and $I$ is the minimum size of the AS that fulfills the reliability requirement, $I=\left|\mathscr{A}_{u}\right|$.

One of the practical implementations to capture the behavior of $Q_{u, c}(n)$ is based on averaging of the instantaneous measurements [21]. The averaging scheme is an Infinite Impulse Response (IIR) filtering:

$$
\widetilde{Q}_{u, c}(n)=a \cdot M_{u, c}^{\mathrm{dB}}(n)+(1-a) \cdot \widetilde{Q}_{u, c}(n-1),
$$

where $a$ is the averaging coefficient which is defined as a function of measurement sampling period $T_{\mathrm{S}}$ and time constant of the IIR filter $T_{0}$; that is,

$$
a=1-0.5^{T_{S} / T_{0}} .
$$

The level of measurement fluctuation with averaging is primarily controlled by the parameter $T_{0}$. Even though a better correlation to $Q_{u, c}(n)$ is achieved by averaging instantaneous measurements, it has shortcoming because it induces delay in AS management procedures. Figure 2 shows comparison 


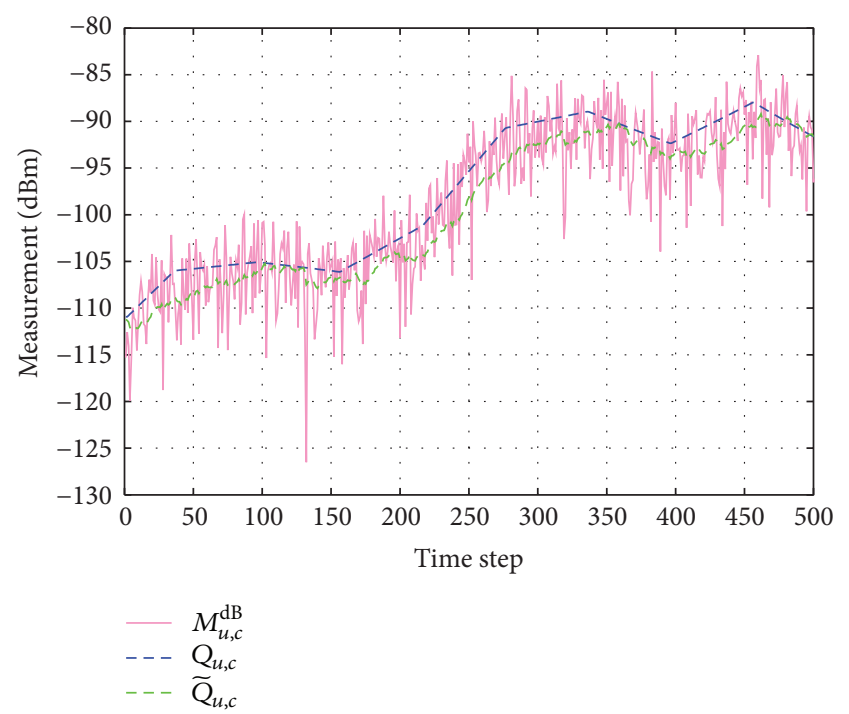

FIGURE 2: Comparison between instantaneous measurement $M_{u, c}^{\mathrm{dB}}$, ideal slow changing channel measurement $Q_{u, c}$, and averaged measurement $\widetilde{Q}_{u, c}$ as a function of time step.

of the instantaneous UE measurement $M_{u, c}^{\mathrm{dB}}$ for a sample cell with the ideal slow changing channel measurement $Q_{u, c}$ and the averaged measurement $\widetilde{Q}_{u, c}$ with time constant $T_{0}$ of $0.1 \mathrm{~s}$. It is shown that the averaged measurement $\widetilde{Q}_{u, c}$ captures the behavior of the ideal slow changing channel measurement $Q_{u, c}$ but with a delay that can create delay in AS management procedures.

Theoretical investigations of the channel characteristics and prediction of $Q_{u, c}(n)$ for achieving ultra-reliable AS management procedures are an interesting area for future research. However, the methodology to capture the nonstationary behavior of $Q_{u, c}(n)$ and the tradeoff between delay and measurement distortion would be a challenge. Herein, this work focuses on the use of averaged measurement $\widetilde{Q}_{u, c}$ to build ultra-reliable AS management procedure. With the help of numerical assessment of the scenario in Section 7, feasible solution that achieves the target in (17) is derived. The performance of the proposed solution will be compared, in Section 8.2.1, to an upper bound that assumes the ideal slow changing channel measurement $Q_{u, c}(n)$.

\section{Derivation of Feasible AS Management Scheme for Multiconnectivity That Uses SFN Transmission}

Feasible AS management procedure for ultra-reliable mobility that is free of connection failures should make sure that the strongest cell in terms of the slow changing channel is included in the AS. For the sake of simplicity, the strongest cell in terms of slow changing channel will be referred to as "ideal strongest."

Having the averaged measurement $\widetilde{Q}_{u, c}$ available, numerical analysis is made to derive AS management procedure that can be used to include the ideal strongest cell into the AS.

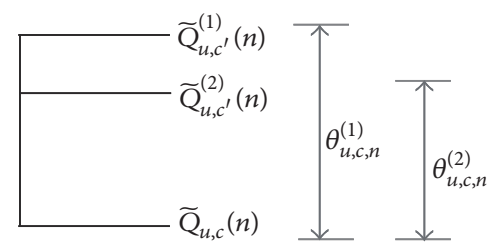

FIGURE 3: Statistics collection for difference between averaged measurement $\widetilde{Q}_{u, c}(n)$ from a cell $c$ and the first strongest averaged measurement $\widetilde{Q}_{u, c^{\prime}}^{(1)}(n)$ for $c \neq c^{\prime}$ and difference between averaged measurement $\widetilde{Q}_{u, c}(n)$ from a cell $c$ and the second strongest measurement $\widetilde{Q}_{u, c^{\prime}}^{(2)}(n)$ for $c \neq c^{\prime}$.

Herein, statistics of the following entities are collected at each time step $n$ from the scenario explained in Section 7.

(i) Difference between averaged measurement $\widetilde{Q}_{u, c}$ of a UE $u$ from certain cell $c$ and averaged measurement of a UE $u$ from the $j$ th strongest cell (in terms of the averaged measurement) excluding the cell $c$ itself: that is,

$$
\begin{aligned}
\Theta^{(j)} & =\left\{\theta_{u, c, n}^{(j)}: \theta_{u, c, n}^{(j)}=\widetilde{Q}_{u, c^{\prime}}^{(j)}(n)\right. \\
& -\widetilde{Q}_{u, c}(n) ; \forall_{u \in \mathscr{U}} ; \forall_{c \in \mathscr{W}} ; c \neq c^{\prime} ; c^{\prime} \in \mathscr{W} ; n \\
& \left.=1,2, \ldots, N_{S}\right\},
\end{aligned}
$$

where $\widetilde{Q}_{u, c^{\prime}}^{(j)}(n)$ is the $j$ th strongest measurement excluding cell $c$ and $N_{S}$ is the total number of simulation time steps. The statistics $\theta_{u, c, n}^{(j)} \in \Theta^{(j)}$ is quantized with a certain granularity resulting in a quantized value which is denoted by $\widetilde{\theta}_{u, c, n}^{(j)}$. Let the set that contains the quantized values $\widetilde{\theta}_{u, c, n}^{(j)}$ be defined as $\widetilde{\Theta}^{(j)}$. For example, Figure 3 demonstrates the statistics collection for the case $j=1$ and 2. It shows the difference between the averaged measurement $\widetilde{Q}_{u, c}(n)$ from cell $c$ and the first strongest averaged measurement $\widetilde{Q}_{u, c^{\prime}}^{(1)}(n)$ for $c \neq c^{\prime}$ and the difference between averaged measurement $\widetilde{Q}_{u, c}(n)$ from a cell $c$ and the second strongest measurement $\widetilde{Q}_{u, c^{\prime}}^{(2)}(n)$ for $c \neq c^{\prime}$.

(ii) Binary indicator that shows whether a cell $c$ is the ideal strongest for $\mathrm{UE} u$ at time step $n$ :

$$
\begin{aligned}
\Upsilon & =\left\{v_{u, c, n}: v_{u, c, n}\right. \\
& =\left\{\begin{array}{ll}
1, & \text { if } c=\underset{l}{\arg \max } Q_{u, l}(n) \\
0, & \text { otherwise; }
\end{array} \forall_{u \in \mathscr{U}} ; \forall_{c \in \mathscr{W}} ; l\right. \\
& \left.\in \mathscr{W} ; n=1,2, \ldots, N_{\mathrm{S}}\right\} .
\end{aligned}
$$




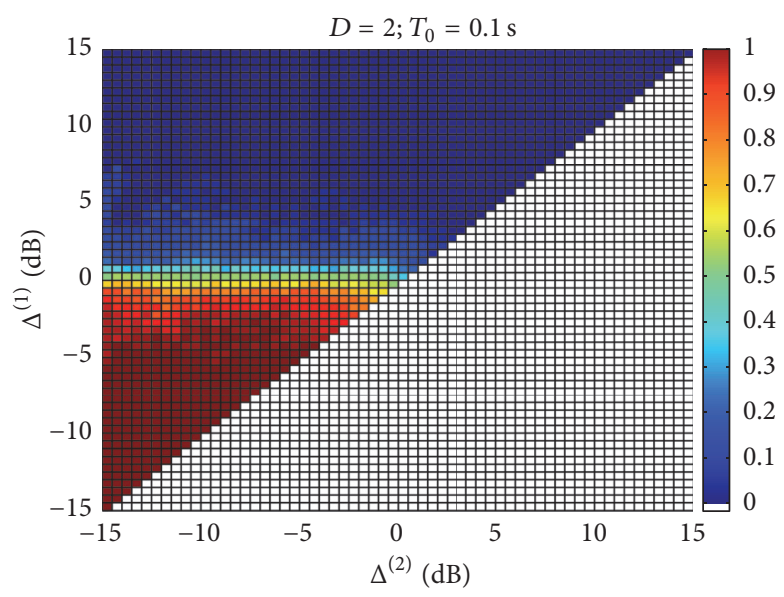

(a)

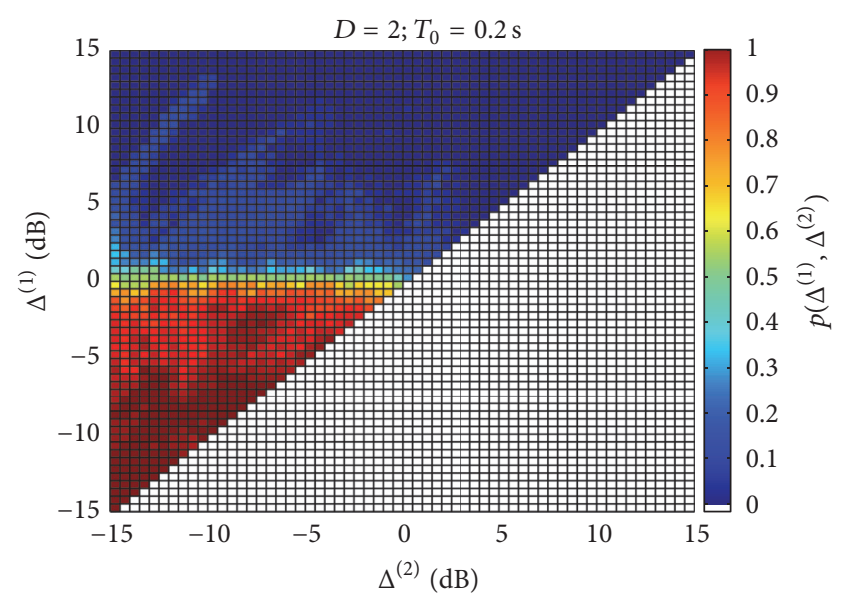

(b)

FIGURE 4: Probability that a cell is the ideal strongest at values $\Delta^{(1)}$ and $\Delta^{(2)}$ for sample diversity order 2 and averaging time constants $0.1 \mathrm{~s}$ and $0.2 \mathrm{~s}$.

The probability that a cell is the ideal strongest is calculated for values $\Delta^{(j)} \in \widetilde{\Theta}^{(j)}$ by counting the corresponding binary indicators in $\Upsilon$. For clarity, it is demonstrated for $j=1$ and 2 . The probability that a cell is the ideal strongest can be calculated for the cases that its averaged measurement is far from the strongest measurement by $\Delta^{(1)}$ and from the second strongest measurement by $\Delta^{(2)}$ as follows:

$$
\begin{aligned}
& p\left(\Delta^{(1)}, \Delta^{(2)}\right) \\
& =\frac{\left|\left\{(u, c, n): \tilde{\theta}_{u, c, n}^{(1)}=\Delta^{(1)} \wedge \widetilde{\theta}_{u, c, n}^{(2)}=\Delta^{(2)} \wedge v_{u, c, n}=1\right\}\right|}{\left|\left\{(u, c, n): \tilde{\theta}_{u, c, n}^{(1)}=\Delta^{(1)} \wedge \tilde{\theta}_{u, c, n}^{(2)}=\Delta^{(2)}\right\}\right|},
\end{aligned}
$$

where $|\{\cdot\}|$ refers to number of elements of the set $\{\cdot\}$.

Figure 4 shows the probability that a cell is the ideal strongest for different values of $\Delta^{(1)}$ and $\Delta^{(2)}$. Two sample averaging time constant parameters, $T_{0}=0.1 \mathrm{~s}$ and $T_{0}=$ $0.2 \mathrm{~s}$, are shown for diversity order of 2 in Figures 4(a) and $4(\mathrm{~b})$, respectively. The white boxes show points that cannot occur because $\Delta^{(1)} \geq \Delta^{(2)}$. With lower values of $\left(\Delta^{(1)}, \Delta^{(2)}\right)$ where a cell is stronger than the first and second strongest average measurements, the probability that a cell is the ideal strongest is higher (shown by dark red color). For example, in Figure 4(a), a cell which is stronger than the first and second strongest averaged measurement by $9 \mathrm{~dB}$ window or more, $\left(\Delta^{(1)}, \Delta^{(2)}\right)<(-9,-9)$, is the ideal strongest cell with probability 1 . Similar analysis of Figure 4 (b) shows that a cell that is stronger than the first averaged measurement by $12 \mathrm{~dB}$ and second averaged measurement by $15 \mathrm{~dB},\left(\Delta^{(1)}, \Delta^{(2)}\right)<$ $(-12,-15)$, is the ideal strongest with probability 1 .

Furthermore, for the case with higher values of $\left(\Delta^{(1)}\right.$, $\left.\Delta^{(2)}\right)$, where a cell is very weak compared to the first and second strongest averaged measurement, the probability that the cell is the ideal strongest is very low. For example, in Figure 4(a), a cell with averaged measurement lower than the strongest averaged measurement by $9 \mathrm{~dB}$ or more,
$\left(\Delta^{(1)}, \Delta^{(2)}\right)>(9,9)$, is the ideal strongest cell with probability 0 .

The above examples show that ultra-reliable AS management scheme that includes the ideal strongest cell can be derived as a set of cells that contain the cell with the strongest averaged measurement as well as cell(s) within a certain window with respect to the strongest average measurement. Accordingly, a procedure that adds cells within a certain window with respect to the strongest averaged measurement ensures that the strongest cell is included with very high probability. Moreover, an AS should be adapted to avoid weak cells because weak cells would lead to lower throughput performance by utilizing resources with a weak signal in the considered SFN transmission. Consequently, a procedure to remove cells should be used to exclude cells with weak signals from the AS. For stable AS management procedures, the AS size should be limited to a certain size, which is maximum AS size. If a candidate neighbor cell is strong enough to be added and the maximum AS size is reached, the candidate neighbor cell can replace the weakest cell in the AS. One of the practical implementations of such AS management procedures is demonstrated in the next section.

\section{Practical AS Management Procedures}

To support the network in the configuration and management of an AS, a UE reports measurements to the network. The measurement reporting mechanism can be periodic or event-triggered. With periodic reporting, the UE sends measurement reports within a certain period configured by the network. In event-triggered measurement reporting, the UE sends measurement report to the network when a certain condition that is configured by the network is fulfilled. This section focuses on event-triggered measurement reporting due to its benefit in reducing signaling overhead compared to periodic measurement reporting. Moreover, it proposes the triggering events defined for 3G Soft Handover in [34] to be 
used for AS management procedures derived in Section 5 for 5 G UDN.

As described in Section 4, the instantaneous measurement of user $u$ for cell $c, M_{u, c}^{\mathrm{dB}}$, can lead to high signaling overhead because the fluctuations from fast fading and noise can cause frequent AS changes. Thus, the UE has to average the measurements before it is used for triggering events. Thus, the measurement report triggering events are based on averaged measurement $\widetilde{Q}_{u, c}$.

6.1. Add Event. The add event has been specified as the " $1 \mathrm{~A}$ " event in [34]. A strong neighbor cell $y$ is added to an AS at time step $n_{0}$ if the following condition is fulfilled:

$$
\begin{aligned}
& \widetilde{Q}_{u, y}(n)>\operatorname{Max}\left(\widetilde{Q}_{u, x}(n)\right)-C_{\text {add }}, \\
& \text { for } n_{0}-T_{\mathrm{T}}<n<n_{0}, x \in \mathscr{A}_{u}, y \notin \mathscr{A}_{u},\left|\mathscr{A}_{u}\right|<K_{\max },
\end{aligned}
$$

where $C_{\text {add }}$ is add offset and $K_{\max }$ is the maximum possible AS size. The above condition checks if the averaged measurement $\widetilde{Q}_{u, y}(n)$ for the neighbor cell $y$ is within the add offset $C_{\text {add }}$ window compared to the strongest cell in the AS. With the expiry of the time to trigger $T_{\mathrm{T}}$, the UE $u$ will send a measurement report to the network and the network adds the strong neighbor cell $y$ to the AS $\mathscr{A}_{u}$.

6.2. Remove Event. The remove event is specified as " $1 \mathrm{~B}$ " event in [34]. Weaker cell(s) is removed from the AS at time $n_{0}$ if the following condition is fulfilled:

$$
\begin{aligned}
& \widetilde{Q}_{u, c}(n)<\operatorname{Max}\left(\widetilde{Q}_{u, x}(n)\right)-C_{\mathrm{rmv}}, \\
& \quad \text { for } n_{0}-T_{\mathrm{T}}<n<n_{0},\{c, x\} \in \mathscr{A}_{u},
\end{aligned}
$$

where $C_{\mathrm{rmv}}$ is the remove offset. A cell is removed if it falls below the strongest cell in the AS by the remove offset $C_{\mathrm{rmv}}$ for a certain time to trigger $T_{\mathrm{T}}$.

In order to avoid the alternating addition and removal of the same cell (similar to a ping-pong), there should be a difference between $C_{\text {add }}$ and $C_{\text {rmv }}$ by a certain offset $q$; that is,

$$
C_{\mathrm{rmv}}=C_{\mathrm{add}}+q .
$$

For example, a cell that is added with an add window $C_{\text {add }}=6 \mathrm{~dB}$ should be removed by a relatively higher remove window; for example, $C_{\mathrm{rmv}}=9 \mathrm{~dB}$ corresponding to $q=$ $3 \mathrm{~dB}$.

6.3. Replace Event. Replace event has also been specified in [34] as "1C" event. When maximum AS size is reached, a strong neighbor cell $y$ replaces the weakest cell $c$ in the AS at time step $n_{0}$ if the following condition is fulfilled:

$$
\begin{aligned}
& \quad\left|\mathscr{A}_{u}\right|=K_{\max }, \\
& \widetilde{Q}_{u, y}(n)>\widetilde{Q}_{u, c}(n)+C_{\text {rplc }}, \\
& \quad \text { for } n_{0}-T_{\mathrm{T}}<n<n_{0}, c \in \mathscr{A}_{u}, y \notin \mathscr{A}_{u},
\end{aligned}
$$

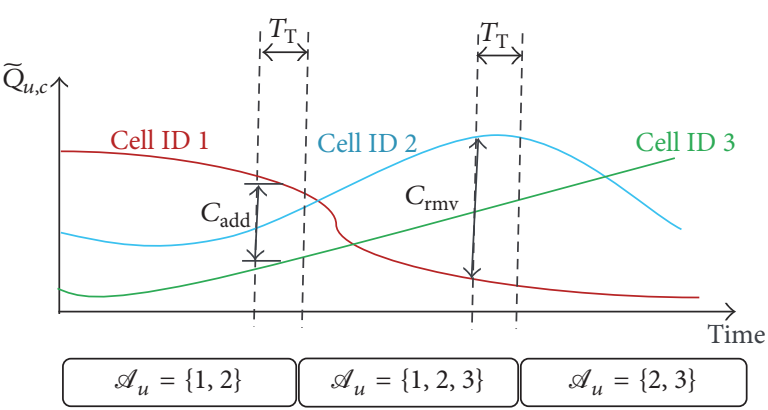

FIGURE 5: Example of AS management procedure for configuring an AS of UE $u, \mathscr{A}_{u}$, based on $3 \mathrm{G}$ trigger events that are reused for $5 \mathrm{G}$ UDN and by using the averaged measurement $\widetilde{Q}_{u, c}$.

where $C_{\text {rplc }}$ is the replace offset. The condition checks if the strong neighbor is better than the weakest cell by the replace offset $C_{\text {rplc }}$.

Figure 5 shows an example of AS management procedure for UE $u$ based on $3 \mathrm{G}$ trigger events that are reused for $5 \mathrm{G}$ UDNs and by using the averaged measurement $\widetilde{Q}_{u, c}$. Assume that the AS of UE $u, \mathscr{A}_{u}$, consists of cell IDs 1 and 2 , and cell ID 3 is a candidate strong cell. Cell ID 3 is added to $\mathscr{A}_{u}$ if the signal strength is within configured add window $C_{\text {add }}$ for certain time steps $T_{\mathrm{T}}$. With movement of the UE, the signal strength from cell ID 1 gets weaker. The UE removes cell ID 1 if the signal strength falls out of the configured remove window $C_{\text {rmv }}$ with respect to the strongest cell.

\section{Scenario and Parameter Settings}

This section describes the scenario and the parameter settings used for performance evaluation of the proposed AS management scheme for 5G UDN deployment.

7.1. Scenario. One of the test cases for future information society is traffic jam which is described as test case 6 in [4]. The users inside vehicles and the vehicles themselves can download capacity demanding services. Moreover, there can be UE at the side of streets inside coffee shops, houses, and so forth that further contribute to the capacity requirements. Standalone UDNs are one of the candidate solutions to satisfy the capacity requirements. Such deployments are also required to cope with mobility challenges when the vehicles are moving fast.

A UDN deployment, shown in Figure 6, is used to emulate the aforementioned scenario based on reasonable parameters for performance evaluation. The network layout has 59 cells with hexagonal layout; the outer most cells are used only to emulate external interference and are not considered in the performance evaluation.

7.2. Simulation Parameters. This subsection demonstrates the parameter settings used for performance evaluation.

It is assumed that the cells in the scenario have an intersite distance (ISD) of $100 \mathrm{~m}$. Each cell transmits at a carrier frequency of $10 \mathrm{GHz}$ and has a bandwidth of $100 \mathrm{MHz}$. The 


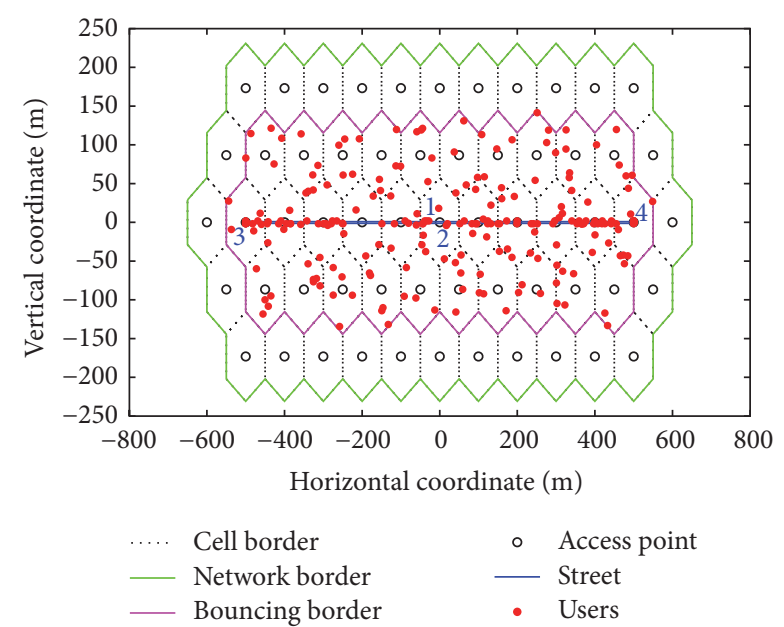

FIGURE 6: Deployment layout for UDN.

bandwidth is split into 10 Physical Resource Blocks (PRBs) where one PRB occupies $10 \mathrm{MHz}$. Besides, two types of UE are considered: street UE that moves at a speed of $60 \mathrm{~km} / \mathrm{h}$ and background UE that moves at speed of $3 \mathrm{~km} / \mathrm{h}$. The street $\mathrm{UE}$ is assumed to be in cars and is randomly distributed on the two-way street. A street UE that reaches the end of a street returns back in the opposite direction on the other side of the road. The mobility of background UE is bounded to the evaluated region whose border is shown by pink lines in Figure 6. Consequently, a UE that reaches the edge of the evaluated region is bounced back. Further details of the parameters related to scenario are listed in Table 1.

The mobility-related simulation parameters are listed separately in Table 2. It includes the values of the parameters described in the system model in Section 3. A static Matlab simulator that runs in terms of time steps is used. The time step is the tradeoff between granularity of capturing mobility events in real time and simulation length to collect robust statistics related to mobility investigations. Taking the aforementioned tradeoff into account, this work assumes a time step of $10 \mathrm{~ms}$ with total number of simulation steps $N_{\mathrm{S}}=2500$. Besides, warm-up time steps $N_{\mathrm{W}}=300$ are considered. The statistics collected from the warm-up time steps are excluded from performance evaluation.

\section{Performance Evaluation}

This section shows the evaluation methodology and simulation results. The simulation result includes comparison of the performance of practical AS management scheme with an upper bound performance and interaction of key parameters in the practical AS management scheme.

8.1. Evaluation Methodology. In this subsection, the key performance indicators that are used for evaluation of multiconnectivity are elaborated. Moreover, a reference singleconnectivity solution that is used as a benchmark for comparison with the proposed multiconnectivity scheme is explained.
TABLE 1: Scenario parameter settings.

\begin{tabular}{lc}
\hline Parameter & Value \\
\hline Network type & UDN \\
ISD & $100 \mathrm{~m}$ \\
Street length & $1 \mathrm{~km}$ \\
Lane size & $3.7 \mathrm{~m}$ \\
Number of lanes & Bidirectional, 1 lane per 1 \\
Total number of street UE pieces & direction \\
Speed of street UE pieces & 102 \\
Total number of background UE pieces & $60 \mathrm{~km} / \mathrm{h}$ \\
Speed of background UE pieces & 155 \\
Transmit power (per 10 MHz) & $3 \mathrm{~km} / \mathrm{h}$ \\
Antenna gain & $30 \mathrm{dBm}$ \\
Antenna height & $5 \mathrm{dBi}$ \\
Path loss model & $10 \mathrm{~m}$ \\
LoS/NLoS correlation distance & ITU-UMi model \\
Shadowing model & $20 \mathrm{~m}$ \\
Shadowing correlation distance & $10 \mathrm{~m}$ \\
Noise power (over 100 MHz) & $-97 \mathrm{dBm}$ \\
\hline Carrier frequency & $10 \mathrm{GHz}$ \\
Bandwidth capacity & $100 \mathrm{MHz}$ \\
1 PRB & $10 \mathrm{MHz}$ \\
Traffic type & Full buffer \\
\hline
\end{tabular}

TABLE 2: Simulation parameter settings.

\begin{tabular}{lc}
\hline Simulation parameter & Settings \\
\hline One simulation time step $T_{\mathrm{S}}$ & $10 \mathrm{~ms}$ \\
Total simulation time steps $N_{\mathrm{S}}$ & 2500 time steps $(25 \mathrm{~s})$ \\
Warm-up time steps $N_{\mathrm{W}}$ & 300 time steps $(3 \mathrm{~s})$ \\
$\mathrm{TTT}$ & $50 \mathrm{~ms}$ \\
Maximum AS size $K_{\max }$ & 5 \\
$q$ & $3 \mathrm{~dB}$ \\
$\mathrm{TH}_{\text {out }}$ & $-8 \mathrm{~dB}$ \\
$\mathrm{TH}_{\text {in }}$ & $-6 \mathrm{~dB}$ \\
$T_{\mathrm{RLF}}$ & 5 time steps $(50 \mathrm{~ms})$ \\
$T_{\text {recovery }}$ & 3 time steps $(30 \mathrm{~ms})$ \\
$\mathrm{TH}_{\text {recovery }}$ & $-8 \mathrm{~dB}$ \\
Time constant $T_{0}$ & $0,0.05,0.1,0.15$, or $0.2 \mathrm{~s}$ \\
Diversity order $D$ & 2,4 , or 8 \\
\hline
\end{tabular}

8.1.1. Radio Link Failures. One of the performance indicators used is the connection failure due to RLFs. The normalized count of RLFs $\widetilde{\xi}_{\text {RLF }}$ is expressed as the ratio of the collected counts of RLFs $\xi_{\mathrm{RLF}}$ (excluding warm-up steps $N_{\mathrm{W}}$ ) to the total number of simulation time steps (expressed in minutes as $\left.\left(10^{-3} \cdot T_{\mathrm{S}}\right) / 60\right)$ and the total number of UE pieces $U$; that is,

$$
\widetilde{\xi}_{\mathrm{RLF}}=\frac{\xi_{\mathrm{RLF}}}{\left(\left(10^{-3} \cdot T_{\mathrm{S}}\right) / 60\right) \cdot\left(N_{\mathrm{S}}-N_{\mathrm{W}}\right) \cdot U} .
$$


8.1.2. AS Updates. AS update could occur when a cell is added, removed, or replaced. With every update in AS, the network sends relevant reconfiguration signaling to the UE concerning the added, removed, or replaced cell. Thus, the number of AS updates gives an indication about the level of signaling. Similar to the previous performance indicator, the normalized count of AS updates $\widetilde{\xi}_{\mathrm{AU}}$ is expressed as the ratio of the collected counts of AS updates $\xi_{\mathrm{AU}}$ (excluding warmup steps $\left.N_{\mathrm{W}}\right)$ to the total number of simulation time steps (expressed in minutes as $\left.\left(10^{-3} \cdot T_{\mathrm{S}}\right) / 60\right)$ and the total number of UE pieces $U$; that is,

$$
\widetilde{\xi}_{\mathrm{AU}}=\frac{\xi_{\mathrm{AU}}}{\left(\left(10^{-3} \cdot T_{\mathrm{S}}\right) / 60\right) \cdot\left(N_{\mathrm{S}}-N_{\mathrm{W}}\right) \cdot U} .
$$

8.1.3. Rapid and Consecutive Add and Remove of Cells. Some of the AS updates occur more frequently leading to signaling overhead. A performance indicator, which is called Consecutive AS Update (CAU), is defined as adding and removing of a cell into AS in a short time or vice versa. In case the measurement is not averaged, unnecessarily frequent AS updates are mainly caused by fluctuation from fast fading and noise. Based on the network operator's demand, the minimum time for which AS updates are CAU can be configured as $T_{\mathrm{CAU}}$. The following cases are considered as CAUs:

(i) A case where a cell is removed immediately after it has been added to the AS of the UE: for example, a cell might be added to the AS of a UE and the same cell is removed within a time window less than $T_{\mathrm{CAU}}$. Such AS updates are considered to be the major source of the signaling overhead associated with AS update configuration.

(ii) A case where a cell is added immediately after it has been removed: for example, a cell is removed after being part of a stable member of AS of a UE. Then, it is added to the AS within a time window less than $T_{\mathrm{CAU}}$.

Similar to the previous performance indicators, the normalized count of CAUs $\widetilde{\xi}_{\mathrm{CAU}}$ is expressed as the ratio of the collected counts of CAUs $\xi_{\mathrm{CAU}}$ (excluding warm-up steps $N_{\mathrm{W}}$ ) to the total number of simulation time steps (expressed in minutes as $\left.\left(10^{-3} \cdot T_{\mathrm{S}}\right) / 60\right)$ and the total number of UE pieces $U$; that is,

$$
\tilde{\xi}_{\mathrm{CAU}}=\frac{\xi_{\mathrm{CAU}}}{\left(\left(10^{-3} \cdot T_{\mathrm{S}}\right) / 60\right) \cdot\left(N_{\mathrm{S}}-N_{\mathrm{W}}\right) \cdot U} .
$$

8.1.4. Cost Function. A cost function $\eta$ is defined for performance evaluation by using the normalized count of RLFs $\xi_{\mathrm{RLF}}$ and the normalized count of CAUs $\xi_{\mathrm{CAU}}$ based on the principles used in [35]. RLFs are considered more critical as compared to CAUs. Thus, it is assumed that the cost function $\eta$ is calculated as the sum of the RLFs and weighted CAUs:

$$
\eta=\widetilde{\xi}_{\mathrm{RLF}}+w_{\mathrm{CAU}} \cdot \widetilde{\xi}_{\mathrm{CAU}}
$$

where $0 \leq w_{\mathrm{CAU}} \leq 1$ is the cost weight associated with CAUs.
8.1.5. Throughput. The throughput of UE is evaluated in terms of 5-\%ile throughput, 50-\%ile throughput, and average throughput. It is based on the full buffer traffic assumption as stated in the system model in Section 3.

8.1.6. Reference for Performance Comparison. Performance of the proposed multiconnectivity scheme that uses SFN transmission is compared with that of a single-connectivity solution that uses classical intracloud handover procedure with hysteresis of $3 \mathrm{~dB}$. The performance indicators used for single connectivity are normalized count of connection failures due to RLFs and handover failures, normalized count of ping-pongs, cost function, number of successful handovers, 5-\%ile throughput, 50-\%ile throughput, and average throughput. The cost function in single connectivity is considered in a similar way to (30) and is calculated as the sum of connection failures and a weighted number of pingpongs. The ping-pong time and the cost weight of ping-pongs are assumed to be the same as $T_{\mathrm{CAU}}$ and weight of CAUs $w_{\mathrm{CAU}}$, respectively.

8.2. Simulation Results. This section presents the simulation results. First, performance of the practical AS management scheme which is discussed in Section 6 is compared with an upper bound performance that uses ideal slow changing channel measurement $Q_{u, c}$. Then, the impact of averaging measurements and diversity order on the practical AS management scheme is shown. For the sake of simplicity, the add/remove window is represented by just the add window $C_{\text {add }}$ in the rest of the paper because the remove window $C_{\mathrm{rmv}}$ is inherently $C_{\text {add }}+q$ as described in Section 6.2.

8.2.1. Comparison of Feasible AS Management Scheme with Upper Bound in terms of RLF. This subsection shows comparison of the performance of the practical AS management scheme with an upper bound performance that assumes ideal slow changing channel measurement $Q_{u, c}$ for AS management.

Figure 7(a) shows the probability that the ideal strongest cell is in the AS of UE and Figure 7(b) shows the normalized count of RLFs $\widetilde{\xi}_{\text {RLF }}$. The upper bound (shown with green curve and "+" marker) ensures that the strongest cell is in the AS with probability 1 . Consequently, there are no RLFs with the upper bound as shown in Figure 7(b). In this case, the add/remove window $C_{\text {add }}$ does not play a role.

For the case with the averaged measurement $\widetilde{Q}_{u, c}$, the normalized count of RLFs $\widetilde{\xi}_{\text {RLF }}$ and the probability that the ideal strongest cell is in the AS of UE are shown for sample parameters $T_{0}=0.1$ and $0.2 \mathrm{~s}$ and $D=2$ and 4 . At lower add/remove window (e.g., $C_{\text {add }}=0 \mathrm{~dB}$ ) and higher averaging time constant (e.g., $T_{0}=0.2 \mathrm{~s}$ ), the probability that the ideal strongest cell is in the AS is lower. This in turn shows higher normalized count of RLFs. Furthermore, it is shown that, with higher add/remove window (e.g., $C_{\text {add }}=9$ or $12 \mathrm{~dB}$ for $T_{0}=0.1 \mathrm{~s}$ ), the ideal strongest cell is included with very high probability which in turn reduces RLFs. Consequently, 


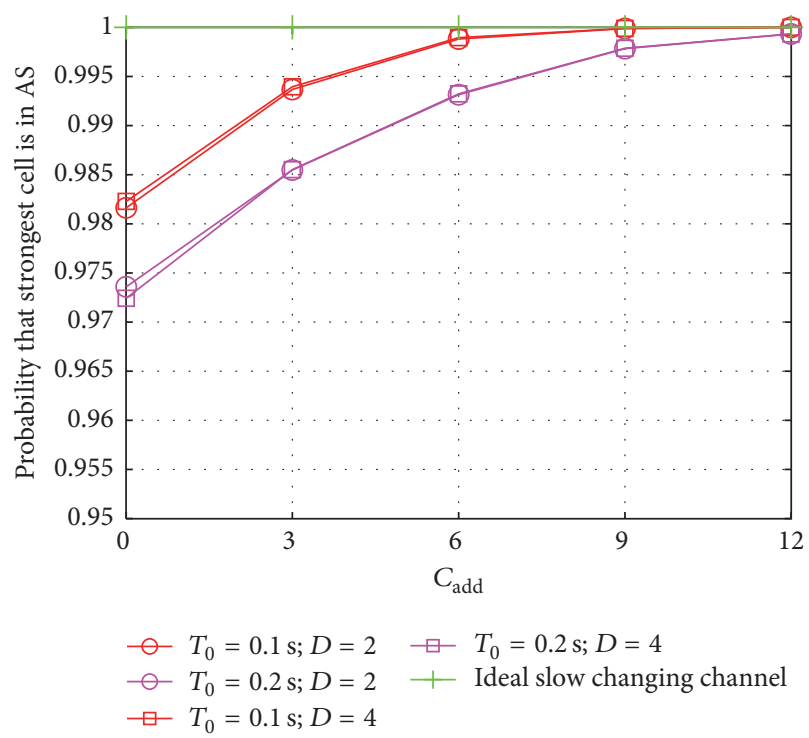

(a)

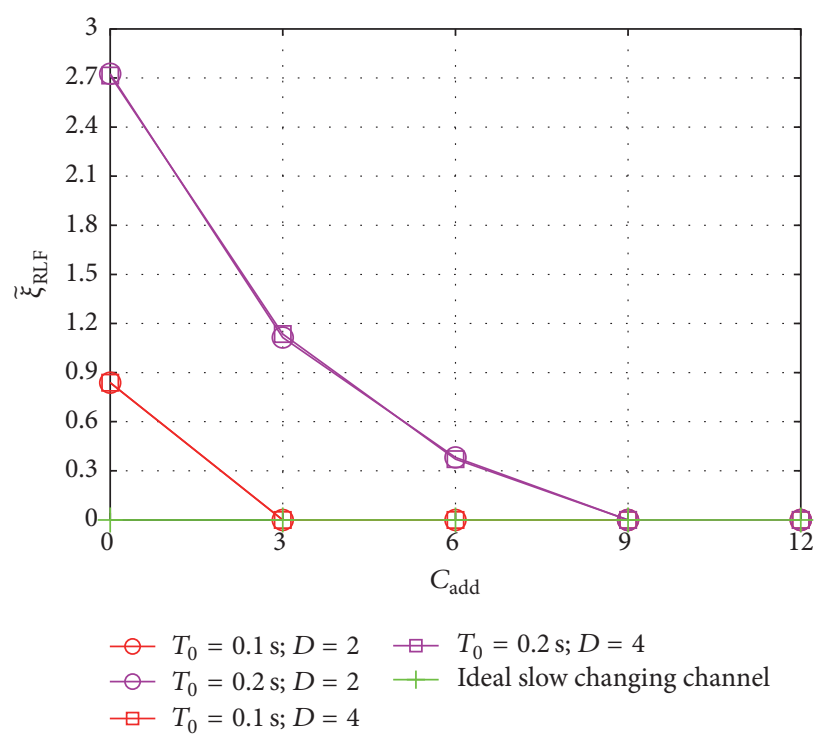

(b)

FIGURE 7: The probability that the strongest cell is in AS, and comparison of normalized RLFs $\tilde{\xi}_{\text {RLF }}$ in the practical AS management scheme with an upper bound.

the performance of the upper bound in terms of RLFs can be achieved by using the proposed multiconnectivity scheme.

\subsubsection{Impact of Averaging Measurements and Diversity Order.} UE measurement processing is one of the critical procedures for a good mobility performance. In this subsection, averaging measurements and UE diversity order are evaluated with the proposed AS management procedure. The parameters $T_{\mathrm{CAU}}$ and $w_{\mathrm{CAU}}$, discussed in Section 8.1.3, can be configured by the network operator based on the requirement of the signaling overhead. In this work, example configurations of $T_{\mathrm{CAU}}=1.5 \mathrm{~s}$ and $w_{\mathrm{CAU}}=20 \%$ are considered.

Figures 8(a), 8(b), and 8(c) show normalized count of $\operatorname{RLFs} \widetilde{\xi}_{\text {RLF }}$, normalized count of CAUs $\widetilde{\xi}_{\text {CAU }}$, and cost function $\eta$, respectively. It is shown for a UE measurement without averaging $\left(T_{0}=0\right)$ for diversity order $D=2$, 4, or 8 as a function of add/remove window $C_{\text {add }}$. For the sake of showing detailed plots, the scale of the vertical axes in Figure 8 is not the same. It is observed that, with the increase of add/remove window $C_{\text {add }}$, the normalized count of RLFs $\widetilde{\xi}_{\text {RLF }}$ declines whereas the normalized count of CAUs $\widetilde{\xi}_{\text {CAU }}$ increases considerably. Consequently, the overall cost function is higher with increase of add/remove window in the absence of averaging. Moreover, the cost function is considerably higher for lower diversity orders because for lower diversity order and no averaging $\left(T_{0}=0 \mathrm{~s}\right)$ the fluctuation of the measurement is higher. The optimal configuration in this particular configuration without averaging is to have a tighter AS (e.g., $\left.C_{\text {add }}=0\right)$.

Figures 9(a), 9(b), and 9(c) show normalized count of RLFs $\widetilde{\xi}_{\text {RLF }}$, normalized count of CAUs $\widetilde{\xi}_{\mathrm{CAU}}$, and cost function $\eta$, respectively. It is shown for sample diversity order $D=2$ as a function of add/remove window $C_{\text {add }}$ with averaging time constant $T_{0}=0.05,0.1,0.15$, or $0.2 \mathrm{~s}$. Similar to Figure 8, the scale of the vertical axes in Figure 9 is not the same for the sake of showing detailed plots. The normalized count of RLFs $\widetilde{\xi}_{\text {RLF }}$ declines with the increase of add/remove window $C_{\text {add }}$. However, the normalized count of CAUs $\widetilde{\xi}_{\text {CAU }}$ increases with the increase of $C_{\text {add }}$. For a lower add/remove window (e.g., $C_{\text {add }}=0 \mathrm{~dB}$ ), increase of averaging time constant $T_{0}$ results in increase of normalized count of RLFs $\tilde{\xi}_{\text {RLF }}$ because of the higher delay discussed in Section 4. However, the normalized count of CAU $\widetilde{\xi}_{\text {CAU }}$ is considerably reduced.

The minimum cost function with no measurement averaging, obtained at $C_{\text {add }}=0 \mathrm{~dB}$ in Figure 8(c), and with averaging at $C_{\text {add }}=3 \mathrm{~dB}$ in Figure 9(c) can be compared for sample diversity order $D=2$. The comparison shows that the cost function $\eta$ is considerably reduced by averaging measurements.

\subsubsection{Impact of Add/Remove Window on Throughput Perfor-} mance. This subsection presents the impact of add/remove window $C_{\text {add }}$ on performance in terms of 5-\%ile, 50-\%ile, and average throughput. The evaluation is based on the modeling assumption discussed in Section 3. The considered unit of throughput is kilo bit per second (kbps) and the evaluation is demonstrated for sample averaging time constant $T_{0}=0.1$ and $0.2 \mathrm{~s}$ and sample diversity order $D=4$.

Figure 10(a) shows the 5-\%ile throughput as a function of add/remove window $C_{\text {add }}$. 5-\%ile throughput represents the performance of cell-edge UE. It is observed that, with the increase of the add/remove window, the 5-\%ile throughput increases due to the gain in SINR from SFN transmission as described in Section 3.2. However, at extremely high add/remove window $C_{\text {add }}$, the 5-\%ile throughput declines as compared to the achieved maximum 5-\%ile throughput. The 

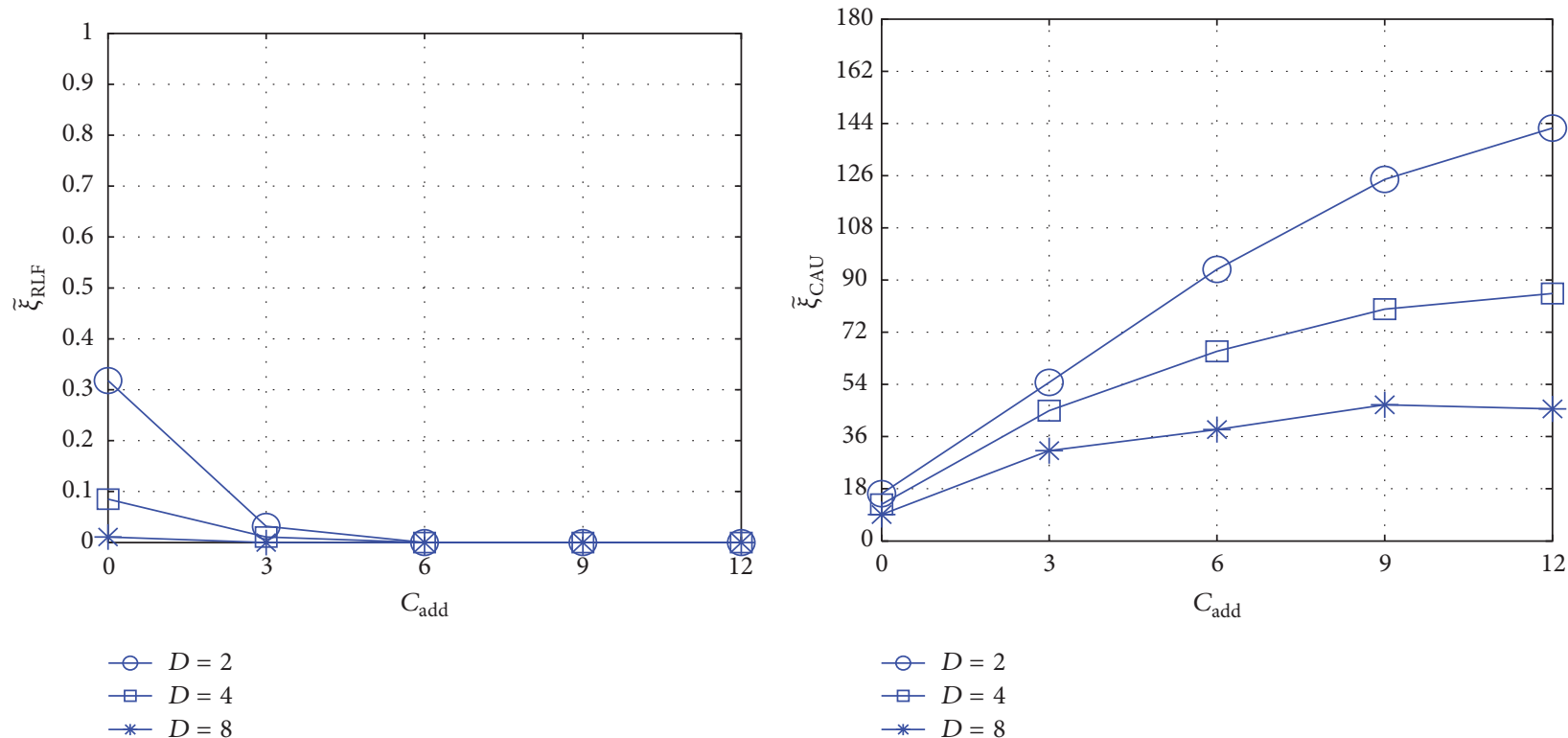

(a)

(b)

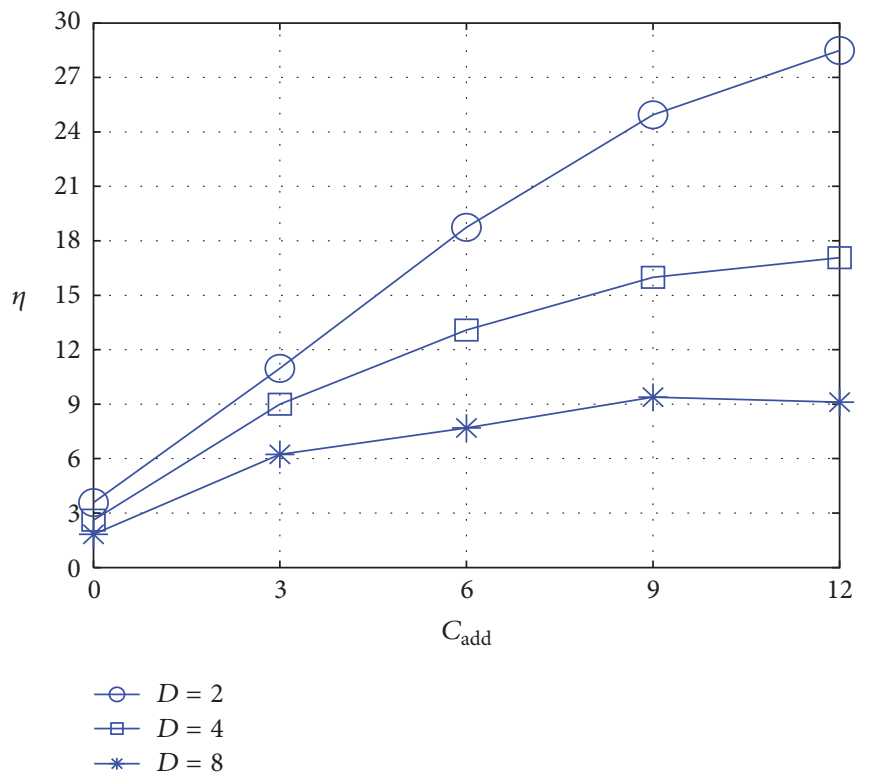

(c)

FIGURE 8: Normalized count of RLFs $\widetilde{\xi}_{\text {RLF }}$, normalized count of CAUs $\widetilde{\xi}_{\text {CAU }}$, and cost function $\eta$ as a function of add/remove window $C_{\text {add }}$ with diversity order $D$ as parameter for averaging time constant $T_{0}=0$.

reason is that, at higher add/remove window, AS size becomes bigger and the number of radio resources blocked by a user increases, as discussed in Section 3.3.

Similarly, the 50-\%ile and average throughput are shown in Figures 10(a) and 10(b), respectively. It is observed that, with initial increase of the add/remove window $C_{\text {add }}, 50-\%$ ile and average throughput slightly increase due to gain in SINR from SFN transmission. However, the 50-\%ile and average throughput decline considerably at extreme settings of $C_{\text {add }}$.

8.2.4. Comparison with Single Connectivity. This subsection demonstrates comparison of the proposed multiconnectivity scheme against a single connectivity that is described in Section 8.1.6. Figures 11(a) and 11(b) compare the proposed multiconnectivity, denoted by $\mathrm{MC}$, and a reference single connectivity, denoted by SC. The comparison is made in terms of the optimal performance in connection failures and the corresponding cost function $\eta$ which can be read from Figures 8 and 9. Furthermore, the comparison is shown for all UE (shown by a set of blue bars), background UE (shown by a set of yellow bars), and street UE (shown by a set of red bars) for different diversity order $D$. As expected, most of the connection failures stem from fast street UE, and it is shown that, with the envisioned multiconnectivity 


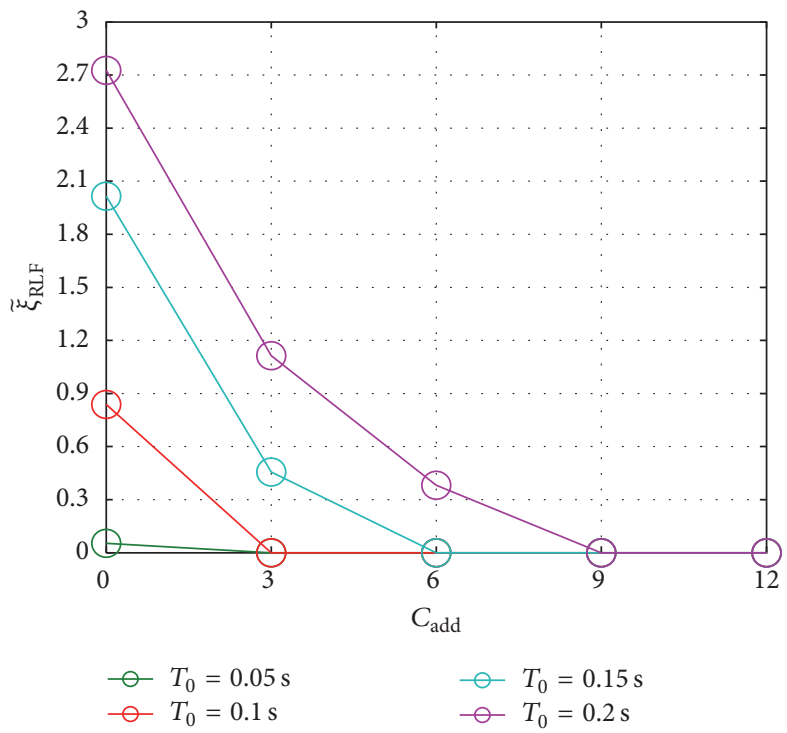

(a)

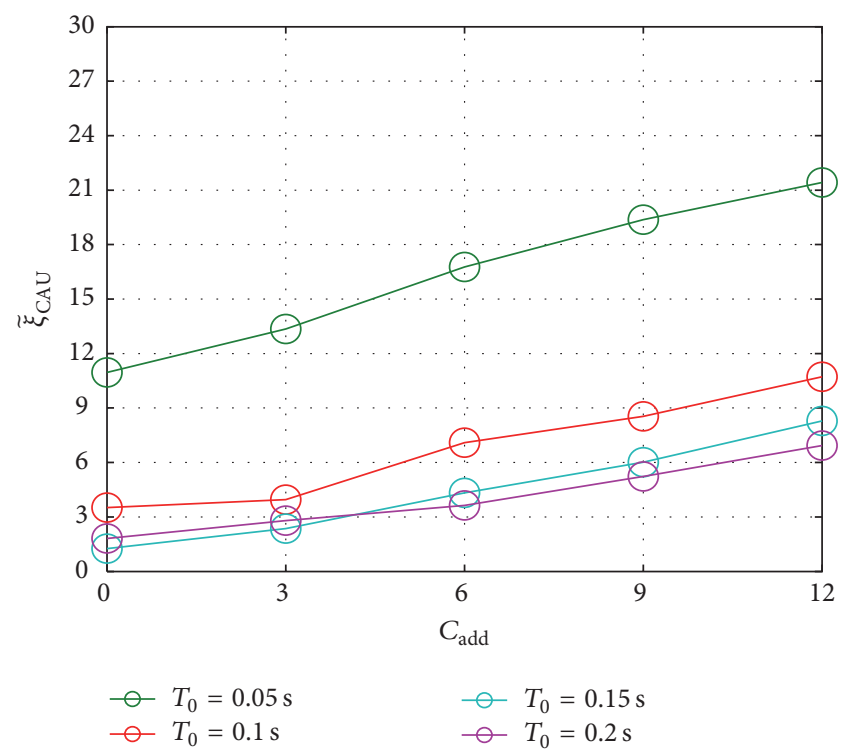

(b)

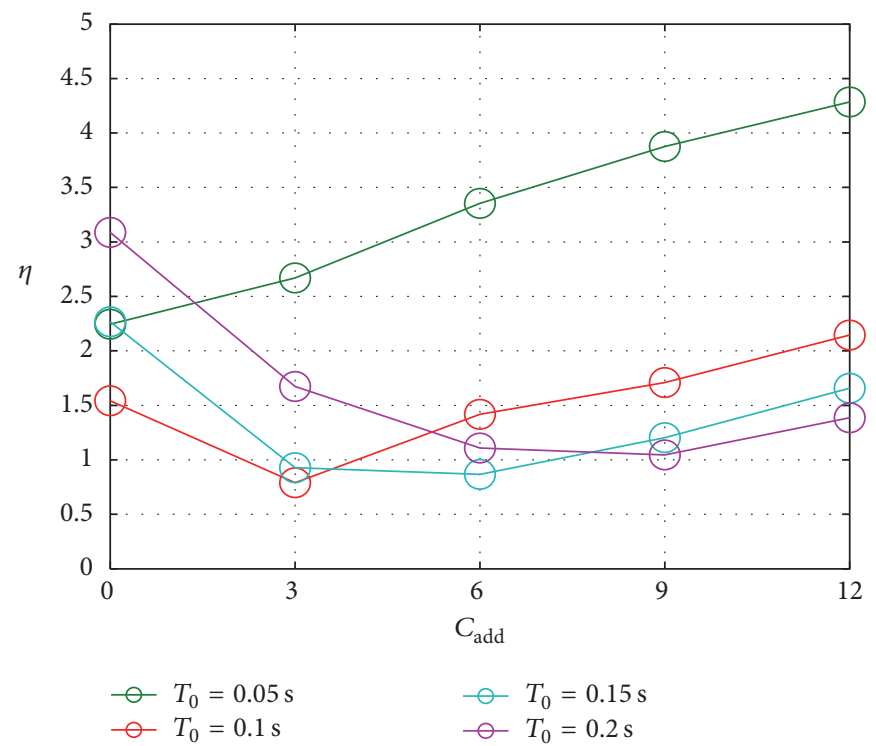

(c)

FigURE 9: Normalized count of RLFs $\widetilde{\xi}_{\text {RLF }}$, normalized count of CAUs $\widetilde{\xi}_{\text {CAU }}$, and cost $\eta$ as a function of add/remove window $C_{\text {add }}$ for diversity order $D=2$ with averaging time constant $T_{0}$ as parameter.

scheme, connection failures are fully resolved. Moreover, the corresponding cost function $\eta$ of all UE in multiconnectivity is reduced by around $80-90 \%$ as compared to that for single connectivity. The major reason is that multiconnectivity gives the required robustness by using AS management scheme that includes the dominant cell in the AS earlier and by the gain on the SINR of control signals. The gain in SINR of the control signals comes from SFN transmission as described in Section 3.2.

Figure 12 compares the overall update of serving cell(s) between multiconnectivity, denoted by MC, and single connectivity, denoted by SC. It is demonstrated with normalized count of AS updates $\widetilde{\xi}_{\mathrm{AU}}$. The comparison is shown for all UE (shown by a set of green bars), background UE (shown by a set of yellow bars), and street UE (shown by a set of red bars) for different diversity order $D$. The normalized count of AS updates for single connectivity refers to the normalized count of successful handovers as described in Section 8.1.6. It is shown that the normalized count of AS updates is considerably high as compared to single connectivity. The major reason is that multiconnectivity is not as conservative in changing serving cell(s) as single connectivity; that is, with multiconnectivity neighbors can be added as member of serving cell(s) even though it is not better than the strongest cell.

Figure 13 shows comparison of multiconnectivity and single connectivity in terms of 5-\%ile, 50-\%ile, and average throughput. With multiconnectivity (which can be read from 


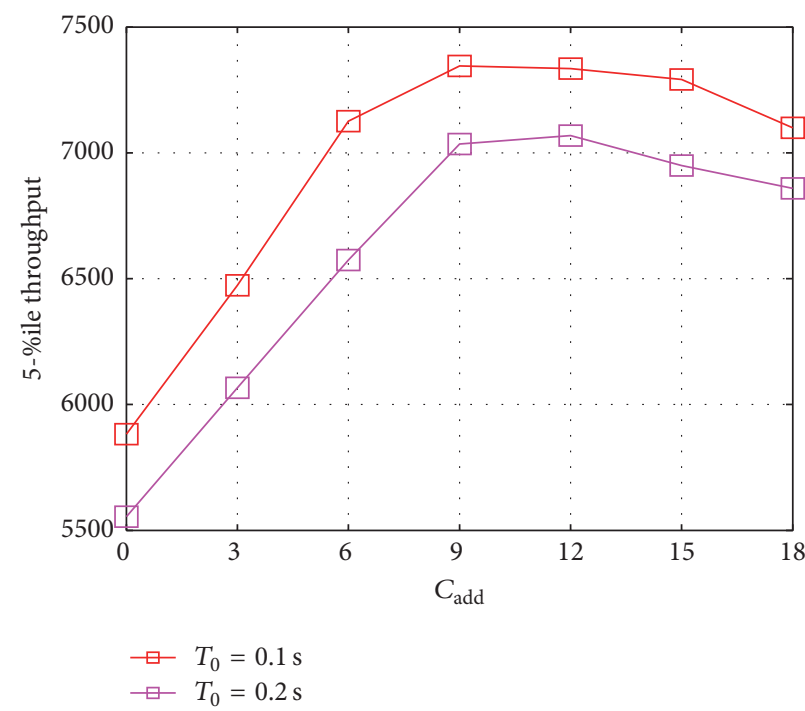

(a)

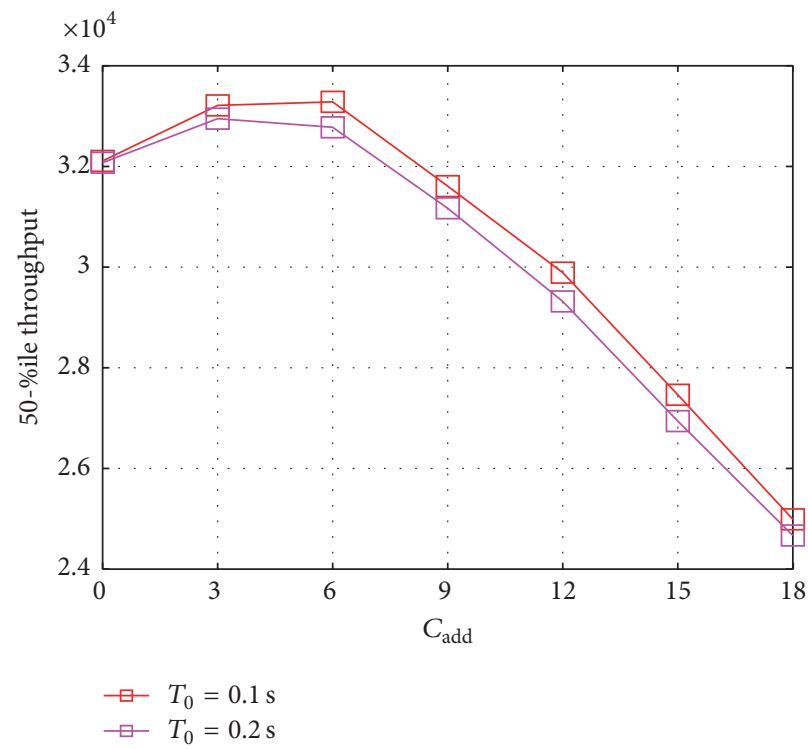

(b)

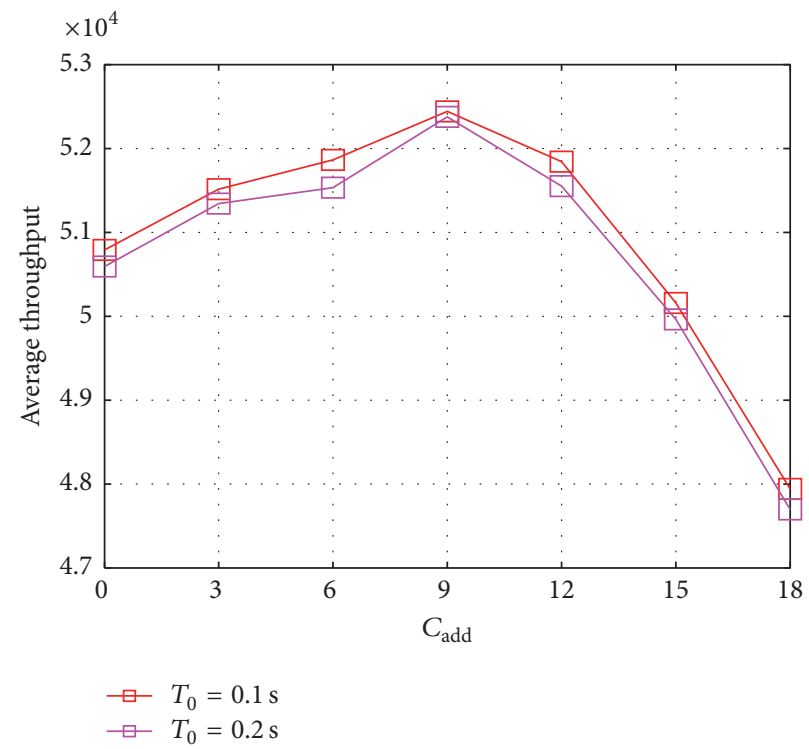

(c)

Figure 10: Performance indicators 5-\%ile throughput, 50-\%ile throughput, and average throughput in kbps as a function of add/remove window $C_{\text {add }}$ for sample averaging time constant $T_{0}=0.1$ and $0.2 \mathrm{~s}$ with diversity order $D=4$.

Figure 10) considering all UE in the network for sample diversity order $D=4$ at parameters $T_{0}=0.1 \mathrm{~s}$ and $C_{\text {add }}=$ $9 \mathrm{~dB}, 5-\%$ ile throughput is boosted by around $43 \%$ because of SFN gain on the data signals. However, there is no significant benefit on the $50-\%$ ile and average throughput.

\section{Conclusion and Outlook}

Driven by the change in demands of the future information society, 5G mobile networks are expected to emerge with technologies that cope with certain requirements. Some of the requirements are capacity and ultra-reliable communication that is free of connection failures. One of the candidate technologies for boosting capacity is UDN. Standalone UDN is prone to handover procedures and connection failures that create service interruptions. This paper proposes low layer multiconnectivity scheme with SFN transmission as a solution to avoid the aforementioned service interruption. The interruptions due to handovers are inherently resolved by preparation of the set of serving cell(s) before the transmission is broken. Furthermore, it is shown that the gain in signal quality of the control signals, due to the SFN transmission, gives increased robustness that is free of RLFs. Besides, the gain in the signal quality of the data signal, due to SFN transmission, gives extra benefit to the cell-edge UE in terms of throughput. 


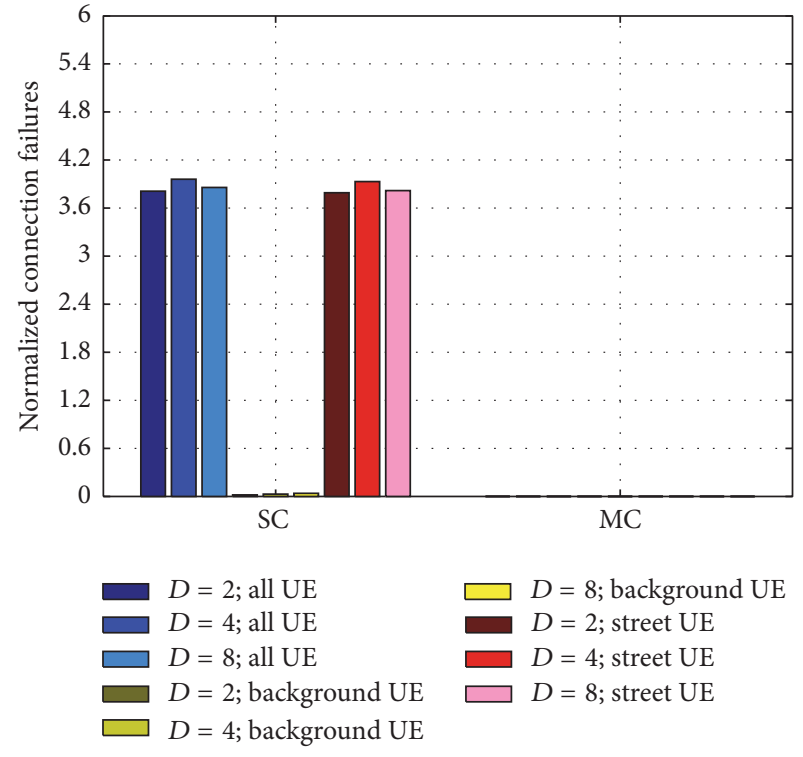

(a)

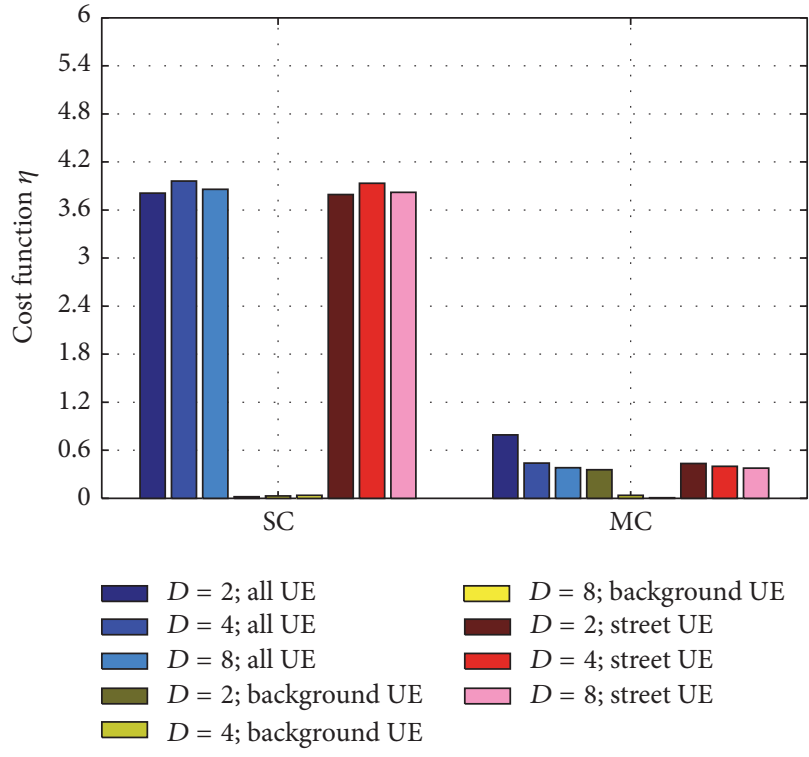

(b)

FIGURE 11: Comparison of multiconnectivity (MC) and single connectivity (SC) in terms of the optimal normalized connection failures and the corresponding cost function $\eta$ for all UE, background UE, street UE, and diversity order $D=2,4$, and 8 .

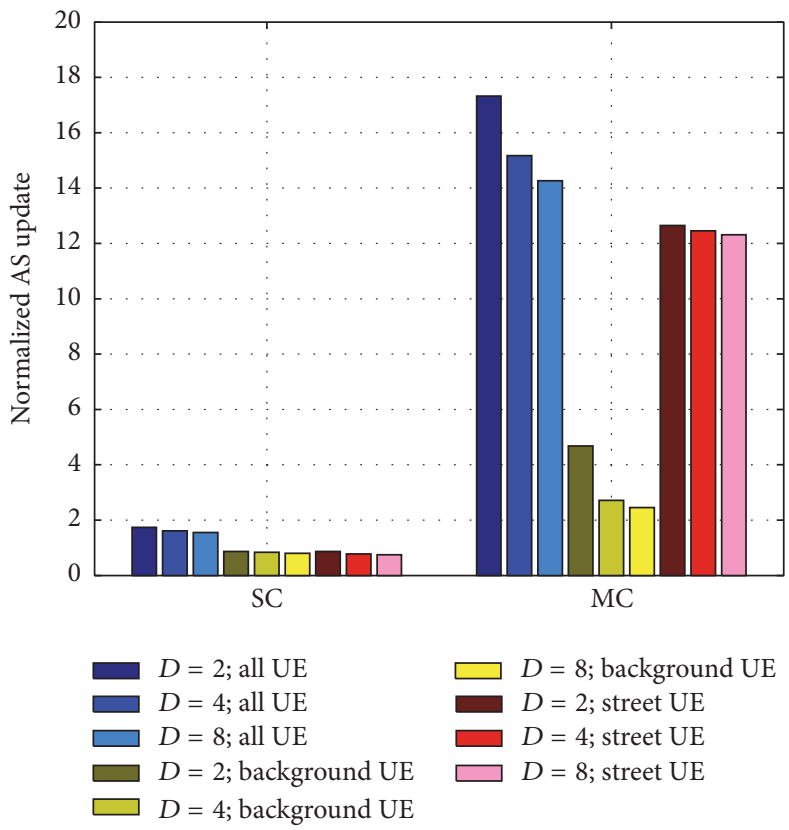

FIGURE 12: Comparison of multiconnectivity (MC) and single connectivity (SC) in terms of normalized count of AS updates $\widetilde{\xi}_{\mathrm{AU}}$ for all UE, background UE, street UE, and diversity order $D=2,4$, and 8 .

The problem formulation in AS management is elaborated and feasible AS management scheme is derived. Practical implementation of the derived AS management scheme was proposed by revisiting prior arts. Then, performance of the practical AS management scheme is compared with an upper bound performance that assumes the ideal slow changing channel measurement. Moreover, key parameters of the practical AS management scheme are evaluated with an elaborated mobility scenario that has mix of slow and fast UE. The finding is that the upper bound performance in terms of RLFs can be achieved by the practical AS management scheme. Furthermore, it is shown that the practical AS management scheme can achieve better performance by averaging measurements with the right configuration. Besides, comparison of the proposed multiconnectivity scheme against single connectivity shows that the envisioned multiconnectivity 


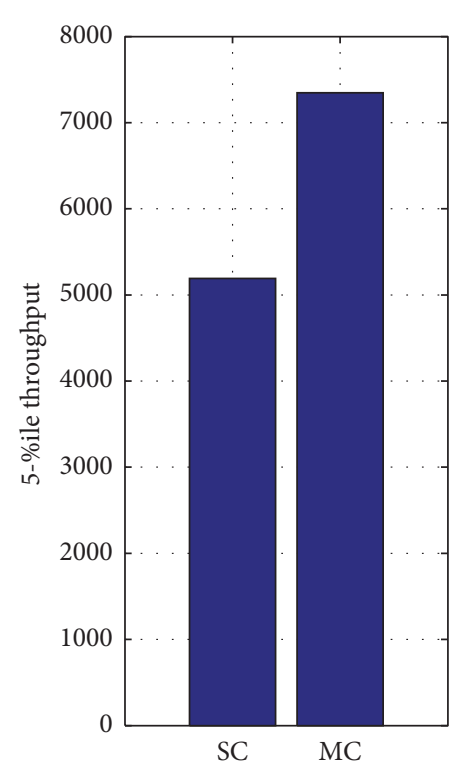

(a)

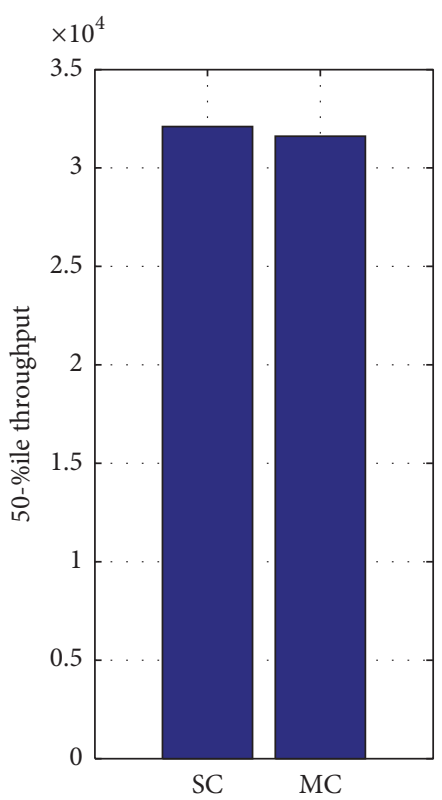

(b)

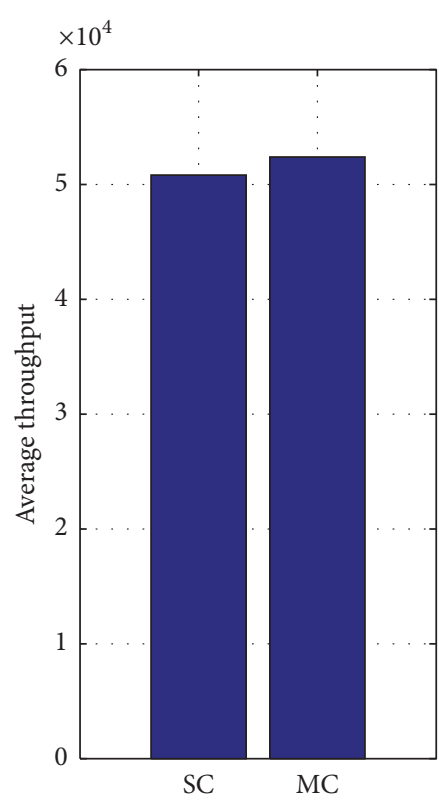

(c)

FIGURE 13: Comparison of multiconnectivity (MC) and single connectivity (SC) in terms of 5-\%ile throughput, 50-\%ile throughput, and average throughput for sample diversity order $D=4$.

scheme has a considerable gain in terms of connection failures and cell-edge throughput.

The transmission scheme in this paper focused on SFN transmission of both control and data signals from the cells of an AS. For future work, further investigation will be made on alternative transmission schemes for control and data signals. One option to be investigated is fast selection of a serving cell among member cells of an AS for transmission of both data and control signals.

\section{Competing Interests}

The authors declare that they have no competing interests.

\section{Acknowledgments}

The authors would like to thank Andreas Lobinger for technical support.

\section{References}

[1] METIS, "Deliverable D1.1 scenarios, requirements and KPIsfor 5G mobile and wireless system," April 2013, http://cordis.europa .eu/docs/projects/cnect/9/317669/080/deliverables/001-METISD11vlpdf.pdff.

[2] A. Osseiran, F. Boccardi, V. Braun et al., "Scenarios for 5G mobile and wireless communications: the vision of the METIS project," IEEE Communications Magazine, vol. 52, no. 5, pp. 2635, 2014.

[3] S. Chen and J. Zhao, "The requirements, challenges, and technologies for $5 \mathrm{G}$ of terrestrial mobile telecommunication," IEEE Communications Magazine, vol. 52, no. 5, pp. 36-43, 2014.
[4] METIS, Deliverable D6.1 Simulation Guidelines, 2013.

[5] P. Rost, C. Bernardos, A. Domenico et al., "Cloud technologies for flexible $5 \mathrm{G}$ radio access networks," IEEE Communications Magazine, vol. 52, no. 5, pp. 68-76, 2014.

[6] Nokia Networks, "Liquid radio: let traffic waves flow most efficiently," White Paper, 2013.

[7] Alcatel-Lucent, "Light radio network: a new wireless experience," 2012, https://techzine.alcatel-lucent.com/lightradiotmnetwork-new-wireless-experience.

[8] China Mobile, "C-RAN: the road towards green radio access networks," White Paper, 2011, http://labs.chinamobile.com/ cran/wp-content/uploads/CRAN_white_paper_v2_5_EN.pdf.

[9] C. Liu, L. Zhang, M. Zhu, J. Wang, L. Cheng, and G.-K. Chang, "A novel multi-service small-cell cloud radio access network for mobile backhaul and computing based on radio-over-fiber technologies," Journal of Lightwave Technology, vol. 31, no. 17, Article ID 6564455, pp. 2869-2875, 2013.

[10] 3GPP, "Evolved universal terrestrial radio access (E-UTRA); Carrier aggregation; Base Station (BS) radio transmission and reception," Tech. Rep. TR 36.808, 2012.

[11] G. Yuan, X. Zhang, W. Wang, and Y. Yang, "Carrier aggregation for LTE-advanced mobile communication systems," IEEE Communications Magazine, vol. 48, no. 2, pp. 88-93, 2010.

[12] 4G Americas, "LTE carrier aggregation technology development and deployment worldwide," White Paper, 2014.

[13] 3GPP, "Technical specification group radio access network; study on small cell enhancement for (E-UTRA) and (EUTRAN); higher layer aspects (Release 12)," Tech. Rep. TR 36.842, 2013.

[14] A. Zakrzewska, D. Lopez-Perez, S. Kucera, and H. Claussen, "Dual connectivity in LTE HetNets with split control- and userplane," in Proceedings of the IEEE Globecom Workshops (GC Wkshps '13), pp. 391-396, IEEE, Atlanta, Ga, USA, December 2013. 
[15] 3GPP, "Technical specification group radio access network; coordinated multi-point operation for LTE physical layer aspects (Release 11)," Tech. Rep. TR 36.819, 2011.

[16] P. Marsch and G. Fettweis, "Static clustering for cooperative multi-point (CoMP) in mobile communications," in Proceedings of the 2011 IEEE International Conference on Communications (ICC '11), pp. 1-6, IEEE, Kyoto, Japan, June 2011.

[17] F. B. Tesema, A. Awada, I. Viering, M. Simsek, and G. P. Fettweis, "Mobility modeling and performance evaluation of multiconnectivity in 5G intra-frequency networks," in Proceedings of the IEEE Globecom Workshops (GC Wkshps '15), pp. 1-6, San Diego, Calif, USA, December 2015.

[18] H. Holma and A. Toskala, LTE for UMTS OFDMA and SCFDMA Based Radio Access, John Wiley \& Sons, New York, NY, USA, 2009.

[19] M. Anas, F. D. Calabrese, P.-E. Östling, K. I. Pedersen, and P. E. Mogensen, "Performance analysis of handover measurements and layer 3 filtering for UTRAN LTE," in Proceedings of the 18th Annual IEEE International Symposium on Personal, Indoor and Mobile Radio Communications (PIMRC '07), pp. 1-5, Athens, Greece, September 2007.

[20] K. Hiltunen, N. Binucci, and J. Bergstrom, "Comparison between the periodic and event-triggered intra-frequency handover measurement reporting in WCDMA," in Proceedings of the IEEE Wireless Communications and Networking Conference (WCNC '00), vol. 2, pp. 471-475, Chicago, III, USA, September 2000.

[21] 3GPP, "Evolved universal terrestrial radio access (E-UTRA); requirements for support of radio resource management (release 10)," Tech. Rep. TR 36.133, 2014.

[22] T. L. Singal, Wireless Communications, Tata McGraw Hill, 2010.

[23] P. M. Shankar, Fading and Shadowing in Wireless Systems, Springer, Berlin, Germany, 2011.

[24] P. Mogensen, K. Pajukoski, E. Tiirola et al., "Centimeter-wave concept for 5G ultra-dense small cells," in Proceedings of the IEEE 79th Vehicular Technology Conference (VTC '14), pp. 1-6, IEEE, Seoul, Republic of Korea, May 2014.

[25] P. Mogensen, K. Pajukoski, E. Tiirola et al., "5G small cell optimized radio design," in Proceedings of the 2013 IEEE Globecom Workshops (GC Wkshps '13), pp. 111-116, IEEE, Atlanta, Ga, USA, December 2013.

[26] F. B. Tesema, P. Zanier, I. Viering, A. J. Fehske, and G. P. Fettweis, "Simplified scheduler model for SON algorithms of eICIC in heterogeneous networks," in Proceedings of the 20th European Wireless Conference (EW '14), pp. 225-230, VDE, Barcelona, Spain, May 2014.

[27] F. B. Tesema, P. Zanier, I. Viering, A. J. Fehske, and G. P. Fettweis, "Comparison of abstract resource management model for SON algorithm of eICIC with real radio resource management," in Proceedings of the 80th IEEE Vehicular Technology Conference (VTC '14), pp. 1-5, IEEE, Vancouver, Canada, September 2014.

[28] M. Batariere, K. Baum, and T. P. Krauss, "Cyclic prefix length analysis for 4G OFDM systems," in Proceedings of the Vehicular Technology Conference (VTC '04), vol. 1, pp. 543-547, Los Angeles, Calif, USA, September 2004.

[29] P. Mogensen, W. Na, I. Z. Kováes et al., "LTE capacity compared to the shannon bound," in Proceedings of the 2007 IEEE 65th Vehicular Technology Conference (VTC '07), pp. 1234-1238, IEEE, Dublin, Ireland, April 2007.

[30] C. Shannon, Collected Papers, Edited by N. J. A. Sloane, A. D. Wyner, IEEE Press, 1993.
[31] F. Kelly, "Charging and rate control for elastic traffic," European Transactions on Telecommunications, vol. 8, no. 1, pp. 33-37, 1997.

[32] S. Boyd, Convex Optimization, Cambridge University Press, New York, NY, USA, 2004.

[33] 3GPP, "Evolved universal terrestrial radio access (E-UTRA); mobility enhancements in heterogeneous networks (release 11)," Tech. Rep. TR 36.839, 2010.

[34] 3GPP, “Technical specification group radio access network; Radio resource management strategies," Tech. Rep. TR 25.922, 1999.

[35] I. Viering, B. Wegmann, A. Lobinger, A. Awada, and H. Martikainen, "Mobility robustness optimization beyond Doppler effect and WSS assumption," in Proceedings of the 8th International Symposium on Wireless Communication Systems (ISWCS '11), pp. 186-191, Aachen, Germany, November 2011. 


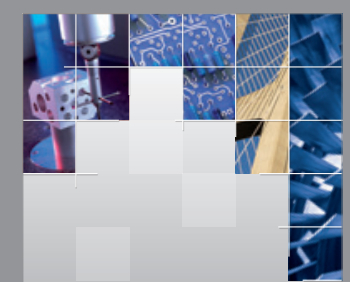

\section{Enfincering}
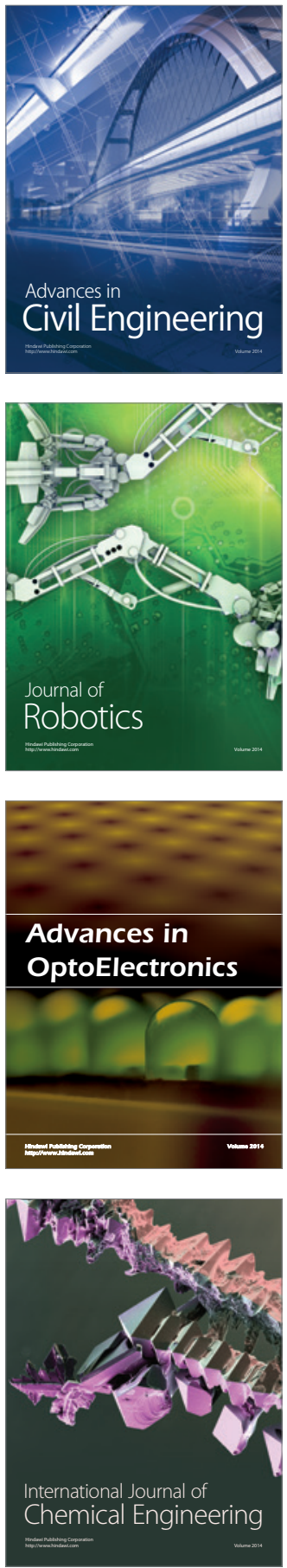

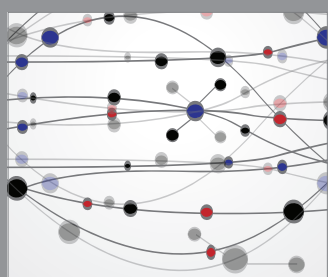

The Scientific World Journal

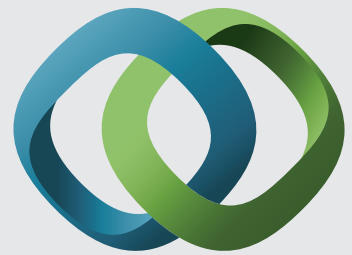

\section{Hindawi}

Submit your manuscripts at

https://www.hindawi.com
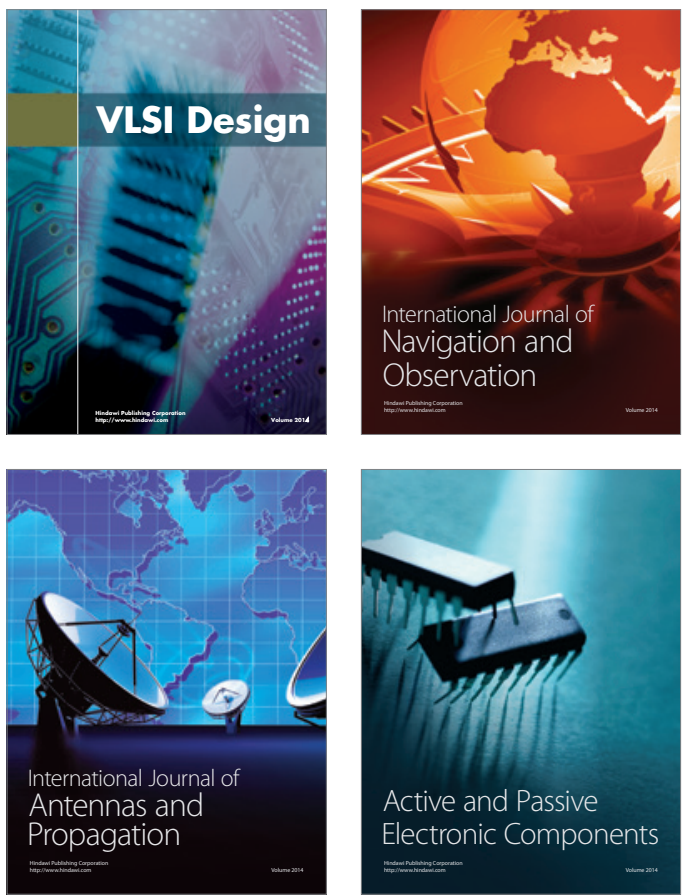
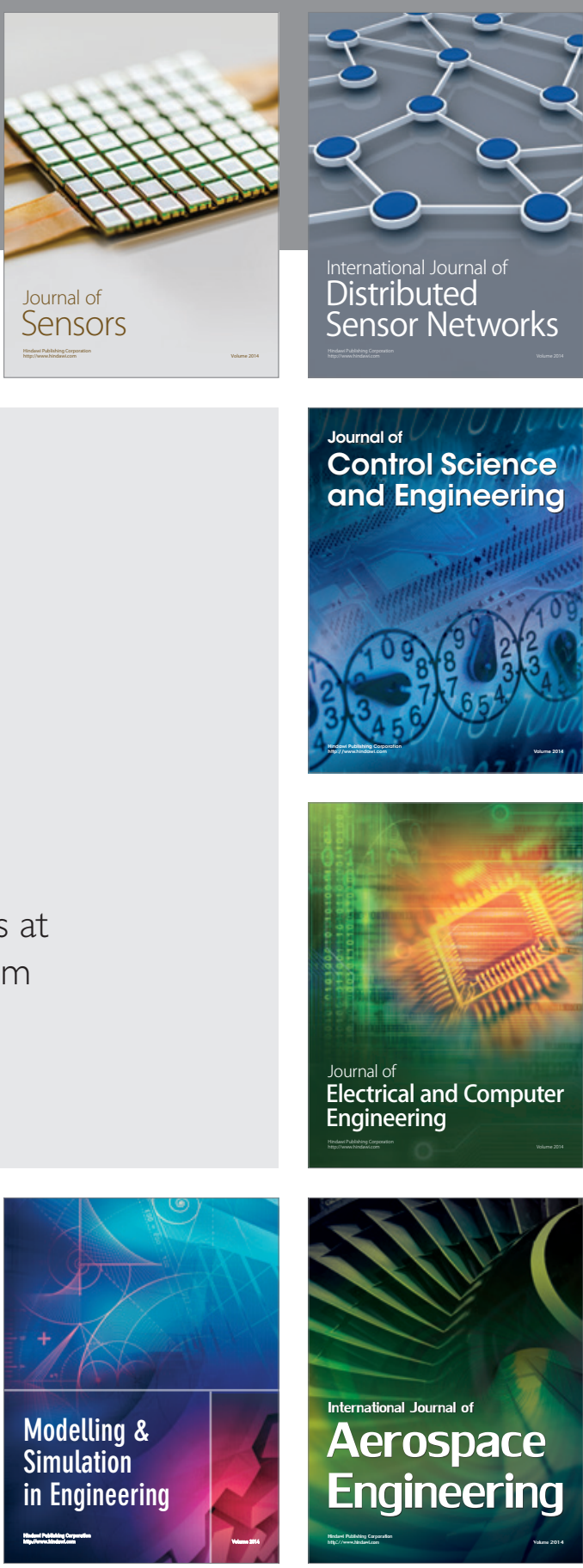

International Journal of

Distributed

Sensor Networks

$-$

Joumal of

Control Science

and Engineering
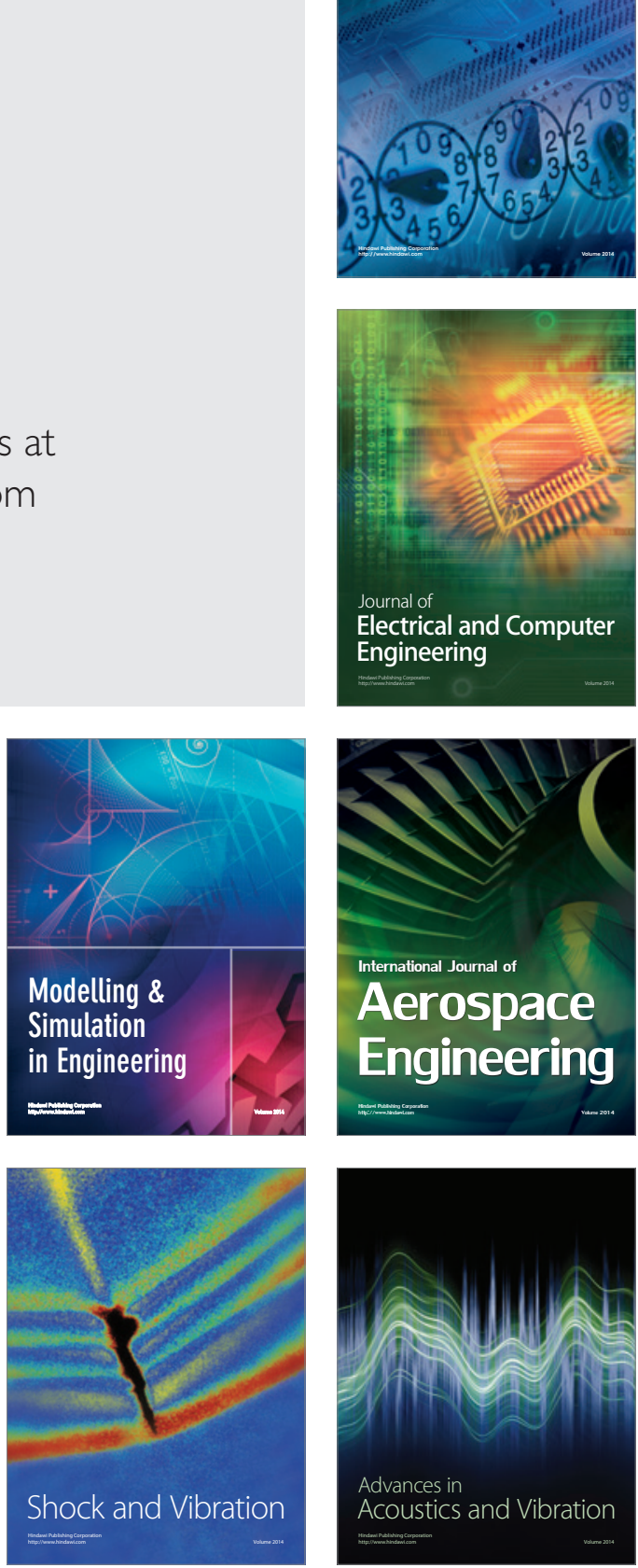